\title{
REGULARITY PROPERTIES OF MILD SOLUTIONS FOR A CLASS OF VOLTERRA EQUATIONS WITH CRITICAL NONLINEARITIES
}

\author{
LUCIANO ABADIAS, EDGARDO ALVAREZ AND CARLOS LIZAMA
}

\begin{abstract}
We study a class of abstract nonlinear integral equations of convolution type defined on a Banach space. We prove the existence of a unique, locally mild solution and an extension property when the nonlinear term satisfies a local Lipschitz condition. Moreover, we guarantee the existence of the global mild solution and blow up profiles for a large class of kernels and nonlinearities. If the nonlinearity has critical growth, we prove the existence of the local $\epsilon$-mild solution. Our results improve and extend recent results for special classes of kernels corresponding to nonlocal in time equations. We give an example to illustrate the application of the theorems so obtained.
\end{abstract}

1. Introduction. Our aim in this article is the study of the following nonlinear Volterra equation of convolution type

$$
u(t)=\int_{0}^{t} a(t-s)\left[A u(s)+f(s, u(s)] d s+u_{0}, \quad t \in[0, T],\right.
$$

where $A$ is a closed linear operator with dense domain $D(A)$ defined on a Banach space $X, a \in L_{\text {loc }}^{1}\left(\mathbb{R}_{+}\right), u_{0} \in X$ and

$$
f: \mathbb{R}_{+} \times X \longrightarrow X
$$

is a continuous function. This class of abstract Volterra integral equations, or equivalent forms of it, arises in early studies of integral equations $[\mathbf{5}, \mathbf{6}]$. In such cases, typically the operator $A$ corresponds

2010 AMS Mathematics subject classification. Primary 34A12, 45D05, 45N05.

Keywords and phrases. Volterra integral equations, local and global, extension and blow up, mild solutions, $\epsilon$-regularity.

The first author was supported by the Universidad de Santiago de Chile, project POSTDOC DICYT-041633LY. The second author was partially supported by Dirección de Investigaciones Universidad del Norte, Project No. 2016-011. The third author was partially supported by CONICYT-Chile under FONDECYT grant No. 1140258 and Anillo ACT 1416.

Received by the editors on November 12, 2016, and in revised form on February 8, 2017. 
to the Laplacian, defined in some smooth domain $\Omega \subset \mathbb{R}^{N}$. One of the main motivations for the analysis of such equations originates by their applicability in the modeling of anomalous diffusion processes, heat conduction with memory and diffusion of fluids in porous media, among other fields of interest $[\mathbf{1 2}, \mathbf{1 7}, \mathbf{2 3}]$.

Note that, for $a(t) \equiv 1$, equation (1.1) is equivalent to the first order nonlinear problem

$$
\left\{\begin{array}{l}
u^{\prime}(t)=A u(t)+f(t, u(t)) \quad t \in(0, T] \\
u(0)=u_{0}
\end{array}\right.
$$

whereas, for $a(t)=t^{\alpha-1} / \Gamma(\alpha), 0<\alpha<1$, strict solutions of (1.1) correspond to mild solutions of the fractional order nonlinear problem

$$
\left\{\begin{array}{l}
D_{t}^{\alpha} u(t)=A u(t)+f(t, u(t)) \quad t \in(0, T], \\
u(0)=u_{0}
\end{array}\right.
$$

where the fractional derivative $D_{t}^{\alpha}$ is understood in Caputo's sense $[3,16]$.

It is worthwhile observing that the linear case of equation (1.1) can be rewritten as the general class of nonlocal in time equations:

$$
\partial_{t}\left(b *\left[u-u_{0}\right]\right)-A u=0,
$$

where $b \in L_{\text {loc }}^{1}\left(\mathbb{R}_{+}\right)$is such that $b * a=1$. See the article [17, Section $1]$, where optimal estimates for the decay in time of solutions in case $A=\Delta$, for the Laplacian operator, has been recently proved.

For the above-mentioned particular cases of kernels $a(t)$, the existence, uniqueness, continuation and regularity properties of locally mild solutions of (1.1) has been studied in a series of recent works, see e.g., the papers $[\mathbf{1}, \mathbf{2}, \mathbf{8}, \mathbf{1 3}, \mathbf{2 2}]$ and the references therein. In reference [8], Andrade et al., have proved sufficient conditions for the existence and uniqueness of a local and global solution to (1.3) and established extension, regularity and comparison results for this solution on Banach spaces as well as an important theorem concerning critical nonlinearities. The study of critical nonlinearities for abstract nonlinear problems of the class (1.2) arises in the paper of Arrieta and Carvahlo [2]. In dealing with this problem, these authors need to understand the scale of fractional powers associated with the linear operator $A$ (being sectorial), especially embeddings into known spaces like $L^{p}$-spaces, and 
study the $\epsilon$-regularity properties of the nonlinearity $f$ in this scale of spaces. This is usually done using Hölder inequality and Sobolev type embeddings. Once the above steps are done, it is necessary to have a regularity theorem establishing that, if $f$ is an $\epsilon$-regular map for some $\epsilon>0$, then we will have existence and uniqueness of $\epsilon$-regular mild solutions.

We observe that existence and uniqueness of the locally mild solution for the initial value problem (1.3) with smoothing effects, as well as estimates for the solution, has been studied by Guswanto and Suzuki [13] by applying the Banach fixed point argument. However, the conditions on the nonlinear term in [13], being general, does not admit critical cases. See also the paper of Roberts [24] for a different approach to the study of blow up of solutions, but whose argument is valid only in the scalar case. Observe that the study of qualitative properties of Volterra equations in the linear and semilinear scalar cases is older, see e.g., the work of Clément and Mitidieri [7] and Friedman [10].

To the best of our knowledge, the study of the existence of locally and globally mild solutions as well as properties of blow up and critical nonlinearities for the class of nonlinear abstract Volterra equation (1.1) are completely absent in the current literature and therefore constitutes an interesting and challenging open problem.

In this article, we succeed in proving the existence of a unique locally mild solution for (1.1) as well as an extension property, when the nonlinear term $f$ satisfies a locally Lipschitz condition. Moreover, we guarantee the existence of a globally mild solution or blow up of maximally local mild solutions. If the nonlinearity has critical growth, we prove the existence of the local $\epsilon$-mild solution.

Concerning methods, Prüss in his book [23] makes a full study for the linear counterpart of the abstract Volterra equation (1.1) based upon the existence of a family of bounded and linear operators which gives a representation of the solution by means of a type of Duhamel's formula. More precisely, the Volterra integral equation

$$
u(t)=\int_{0}^{t} a(t-s) A u(s) d s+f(t), \quad t \geq 0
$$

has a solution if and only if the operator $A$ generates a strongly 
continuous family of bounded and linear operators

$$
(R(t))_{t \geq 0} \subset \mathcal{B}(X)
$$

for (1.4), see [23, Proposition 1.1]. We observe that there is a large volume of literature about operator families that solves abstract linear evolution equations, see for example, $[\mathbf{1 5}, \mathbf{1 9}, \mathbf{2 0}, \mathbf{2 1}]$.

Based on operator theoretical methods and the ideas developed in $[8,2]$, we obtain necessary conditions for the existence and uniqueness of locally and globally mild solutions of (1.1) as well as continuation properties and blow up in finite time. Moreover, assuming that $A$ is a sectorial operator, we are able to show the existence of $\epsilon$-regular mild solutions with spatial regularity in the special case of kernels

$$
a(t)=\frac{t^{\alpha-1}}{\Gamma(\alpha)} e^{-\delta t}, \quad 0<\alpha<1, \delta \geq 0 .
$$

We note that this class of kernels naturally appears in applications since it corresponds to certain viscoelastic materials [23, subsection 5.2].

It is worthwhile mentioning that the results of this paper extend those of Andrade, et al., [8] for the time fractional equation (1.3) and from the paper by Arrieta and Carvahlo [2] for the first order nonlinear problem (1.2). Note that some earlier results in $[\mathbf{4 , 2 5 , 2 6 ]}$ are also included in our analysis.

This paper is organized as follows. Section 2 is devoted to preliminaries, introducing the adequate notion of resolvent families of operators needed in the forthcoming sections (Definition 2.1). By means of this tool, we define the appropriate notion of a mild solution to (1.1) (Definition 2.7). We also recall the notions of a sectorial operator, powers of an unbounded operator and the concept of an $\epsilon$-regular map. This last concept is an interesting tool introduced some years ago by Arrieta and Carvahlo [2] which will be very useful in the study of spatial regularity.

In Section 3, we obtain existence, uniqueness and continuation results to (1.1) when the nonlinear term $f$ is a globally Lipschitz continuous function (Theorem 3.2 and Theorem 3.5). We show an argument to guarantee the existence of global mild solution or the blow up in finite time of maximally local mild solutions (Theorem 3.7).

We observe that the assumption on the nonlinear term $f$ is rather general but it does not allow for the treatment of problems where the 
nonlinearity has critical growth. Hence, we treat the critical case in Section 4, proving the existence of local $\epsilon$-regular mild solutions to (1.1), see Theorem 4.11. Finally, as an application of our results, we ensure the existence of a locally mild solution for an integral equation arising in viscoelasticity theory (Example 4.13).

Finally, we note that existence of local solutions for equation (1.3) in the more general case $A=A(t)$ has recently been studied. For instance, in [22] the existence of a local solution in the case $A(t)=a(t) \Delta$ ( $\Delta$ being the Laplacian operator) is proved by means of an argument using the Leray-Shauder fixed point and Banach contraction mapping theorems. Therefore, it seems to be an interesting future task to study the existence of a local solution and regularity properties for the class of integral equations (1.1) in such a nonautonomous case.

2. Preliminaries. We denote by $\mathcal{B}(X)$ the space of bounded and linear operators from $X$ to $X$. Let $a \in L_{\text {loc }}^{1}\left(\mathbb{R}_{+}\right) \cap C((0, \infty))$ be a scalar kernel and $f: \mathbb{R}_{+} \times X \rightarrow X$ a continuous function. We consider the Volterra equation

$$
u(t)=\int_{0}^{t} a(t-s)\left[A u(s)+f(s, u(s)] d s+u_{0}, \quad t \in J:=[0, T],\right.
$$

where $u_{0} \in X$ and $A$ is a closed linear operator with dense domain $D(A)$ defined on a Banach space $X$. In the sequel, we denote by $[D(A)]$ the domain of $A$ equipped with the graph norm.

A function $u \in C(J ; X)$ is called a solution of $(2.1)$ on $J$ if $u \in$ $C(J ;[D(A)])$ and $(2.1)$ hold on $J$.

We will need the following notion of integral resolvent families.

Definition 2.1. Let $a \in L_{\text {loc }}^{1}\left(\mathbb{R}_{+}\right) \cap C((0, \infty))$ be a scalar kernel. A family $(S(t))_{t>0} \subset \mathcal{B}(X)$ of bounded linear operators in $X$ is called an integral resolvent if the following conditions are satisfied.

(i) $S(t)$ is strongly continuous on $(0, \infty)$ and

$$
\lim _{t \rightarrow 0} \frac{S(t)}{a(t)} x=x, \quad \text { for all } x \in X .
$$

(ii) $S(t) D(A) \subset D(A)$ and $A S(t) x=S(t) A x$ for all $x \in D(A)$ and $t>0$. 
(iii) The equation

$$
S(t) x=a(t) x+\int_{0}^{t} a(t-s) A S(s) x d s \quad \text { for all } x \in D(A), t>0
$$

holds.

The integral resolvent is unique, if it exists. In such a case, we call the operator $A$ the generator of the integral resolvent $(S(t))_{t>0}$. In addition, by Definition 2.1 (iii), we have $(a * S)(t) x \in D(A)$ for all $x \in X$ and $t>0$, since $A$ is closed and $D(A)$ is dense ([23, Proposition 1.1]). Observe that $S(t)$ cannot be defined for $t=0$, but it is locally integrable, i.e.,

$$
\int_{0}^{t_{0}}\|S(t)\| d t<\infty \quad \text { for } t_{0}>0
$$

since $(S(t))_{t>0}$ is strongly continuous and it behaves as $a \in L_{\text {loc }}^{1}\left(\mathbb{R}_{+}\right) \cap$ $C((0, \infty))$ in a neighborhood of zero. This last observation is also a consequence of Remark 2.6, below.

Remark 2.2. Definition 2.1 corresponds to a slight modification to those given by Prüss [23, Definition 1.6], where (i) is replaced by local integrability instead of strong continuity. Observe that the given condition near zero instead of the most common $S(0)=a(0) I$ allows the treatment of singular kernels $a(t)$. See also Li and Peng [18] where this approach has been taken in the context of resolvent families for fractional Cauchy problems of order $0<\alpha<1$.

Remark 2.3. Given $\alpha>0$, we define

$$
g_{\alpha}(t):=\frac{t^{\alpha-1}}{\Gamma(\alpha)}, \quad t>0,
$$

where $\Gamma$ denotes the Gamma function. Observe that $g_{\alpha}$ satisfies the semigroup property: $g_{\alpha} * g_{\beta}=g_{\alpha+\beta}$ where $\alpha, \beta>0$. For the case of $a(t)=g_{\alpha}(t)$, our Definition 2.1 coincides with the concept of $\alpha$ resolvent family given by some authors, see e.g., [13] and the references therein. 
Remark 2.4. Let $a \in L_{\text {loc }}^{1}\left(\mathbb{R}_{+}\right) \cap C((0, \infty))$ be given, and suppose that there exists a $b \in L_{\text {loc }}^{1}\left(\mathbb{R}_{+}\right)$such that

$$
(a * b)(t):=\int_{0}^{t} a(t-s) b(s) d s=1, \quad \text { for all } t>0 ;
$$

then,

$$
R(t) x:=\int_{0}^{t} b(t-s) S(s) x d s, \quad x \in X, t>0,
$$

corresponds to the so-called resolvent family generated by $A$, see [23, Definition 1.3]. Indeed, it is clear that the identity

$$
R(t) x=x+\int_{0}^{t} a(t-s) A R(s) x d s \quad \text { for all } x \in D(A), t>0
$$

holds. Also, $(a * R)(t) x \in D(A)$ for $x \in X$ and $t>0$. In addition, for $x \in X$ and $t>0$, we obtain

$$
\begin{aligned}
\|R(t) x-x\| & =\|A(a * R)(t) x\|=\|A(a * b * S)(t) x\| \\
& =\left\|A \int_{0}^{t} S(s) x d s\right\| \longrightarrow 0, \quad \text { as } t \rightarrow 0 .
\end{aligned}
$$

Thus, we extend $R(0)=I$ by strong continuity. Note that kernels $a$ satisfying (2.3) have been studied in the literature. A wide class of such kernels is provided by the class of creep functions, see [23, Proposition 4.4]. By definition, a creep function is nonnegative, nondecreasing and concave.

Remark 2.5. We note that, in the case of $a(t)=g_{\alpha}(t)$, a resolvent family solves the initial value fractional Cauchy problem

$$
{ }_{C} D^{\alpha} u(t)=A u(t), \quad u(0)=u_{0}, t>0,
$$

where ${ }_{C} D^{\alpha}$ denotes the fractional derivative of order $0<\alpha<1$ in the sense of Caputo. Instead, the corresponding integral resolvent solves the fractional Cauchy problem

$$
{ }_{R} D^{\alpha} u(t)=A u(t), \quad t>0,
$$

with initial condition $\lim _{t \rightarrow 0^{+}}\left(g_{1-\alpha} * u\right)(t)=u_{0}$, where ${ }_{R} D^{\alpha}$ denotes the fractional derivative in the sense of Riemann-Liouville [16]. 
Remark 2.6. Observe that Definition 2.1 (i) together with the strong continuity of an integral resolvent imply that there exist a $t_{0}>0$ and a positive constant $M\left(t_{0}\right)$ such that the estimates

$$
\|S(t) x\| \leq M\left(t_{0}\right) a(t)\|x\|, \quad t \in\left(0, t_{0}\right], x \in X,
$$

and (see equation (2.5))

$$
\|R(t) x\| \leq M\left(t_{0}\right)\|x\|, \quad t \in\left[0, t_{0}\right], x \in X,
$$

hold.

Remark 2.6 will be very useful in several instances of local analysis of solutions for (2.1).

Next, we introduce the appropriate notion of mild solution which will be used throughout this paper.

Definition 2.7 (Mild solution). Let $A$ be the generator of an integral resolvent $(S(t))_{t>0}, f: J \times X \rightarrow X$ a continuous function and $a \in L_{\text {loc }}^{1}\left(\mathbb{R}_{+}\right) \cap C(0, \infty)$ satisfying the condition (2.3). A function $u \in C(J ; X)$ is called a mild solution of equation (2.1) if it satisfies the integral equation

$$
u(t)=R(t) u_{0}+\int_{0}^{t} S(t-s) f(s, u(s)) d s, \quad t \in J .
$$

We shall see in Theorem 3.2 that, under locally Lipschitz assumptions on the function $f$, there exists a unique continuous mild solution, well-defined at $t=0$.

We recall the following notion from operator theory.

Definition 2.8 (Sectorial operator). Let

$$
A: D(A) \subset X \longrightarrow X
$$

be a linear operator. We say that $A$ is sectorial of angle $\theta \in[0, \pi)$ if

$$
\sigma(A) \subset \overline{\Sigma_{\theta}}
$$

and

$$
\left\|\lambda\left(\lambda-A^{-1}\right)\right\| \leq M_{\varphi}, \quad \lambda \in \mathbb{C} \backslash \overline{\Sigma_{\varphi}}, \quad \text { for all } \varphi \in(\theta, \pi),
$$


where $\sigma(A)$ denotes the spectrum of $A$ and

$$
\Sigma_{\theta}:=\{\lambda \in \mathbb{C}: \lambda \neq 0,|\arg (\lambda)|<\theta\}
$$

for $\theta \in(0, \pi]$ and $\Sigma_{0}:=(0, \infty)$ if $\theta=0$.

If $-A$ is closed, densely defined and sectorial of angle $\theta \in[0, \pi / 2)$, then $A$ generates a bounded holomorphic $C_{0}$-semigroup $(T(t))_{t \geq 0}$ on $X$. Also, for each $\varphi \in(\theta, \pi)$, the operator $A$ induces a functional calculus (algebra homomorphism) between the spaces $H_{0}^{\infty}\left(\Sigma_{\varphi}\right)$ and $\mathcal{B}(X)$ given by

$$
f(-A):=\frac{1}{2 \pi i} \int_{\gamma_{\omega}} f(z)(z-A)^{-1} d z, \quad f \in H_{0}^{\infty}\left(\Sigma_{\varphi}\right),
$$

where $H_{0}^{\infty}\left(\Sigma_{\varphi}\right)$ denotes the Banach space of bounded holomorphic functions $f$ with the norm $\|f\|_{\infty, \Sigma_{\varphi}}:=\sup \left\{|f(z)|: z \in \Sigma_{\varphi}\right\}$ such that $f$ is regularly decaying at 0 and at $\infty$. The complex path $\gamma_{\omega}$, with $\omega \in(\pi-\varphi, \pi-\theta)$, is given by

$$
\gamma_{\omega}:=\left\{\lambda e^{i \omega}: \lambda \geq 0\right\} \cup\left\{\lambda e^{-i \omega}: \lambda \geq 0\right\},
$$

oriented counterclockwise. For more details, see [14, Chapter 2].

Let $0<\beta<1$. The above functional calculus allows for the definition of the fractional powers of operator $-A$ in the following manner:

$$
(-A)^{\beta} x:=(I+A)\left(\frac{z^{\beta}}{1+z}\right)(-A) x, \quad x \in D\left((-A)^{\beta}\right),
$$

where $D\left((-A)^{\beta}\right)$ is the space where the above operator is well defined. If $\beta \in(n, n+1)$ with $n \in \mathbb{N}$, then $(-A)^{\beta}:=(-A)^{n}(-A)^{\beta-n}$. For convenience, we denote the fractional spaces

$$
X^{\beta}=D\left((-A)^{\beta}\right)
$$

with the graph norm $\|x\|_{X^{\beta}}=\left\|(-A)^{\beta} x\right\|$. If $\beta \in(n, n+1)$ with $n \in \mathbb{N}$, then $(-A)^{\beta}=(-A)^{n}(-A)^{\beta-n}$. For more information, see [14, Chapter $3]$.

To finish this section of preliminaries, we recall the following definitions which will be used in the main results of the paper. 
Definition 2.9 (Locally Lipschitz function). We say that a function $f:[0, \infty) \times X \rightarrow X$ is uniformly and locally Lipschitz with respect to the first and second variable, respectively, if, for each fixed $x \in X$, there exist an open ball $B=B(x)$ and a constant $L=L(B)>0$ such that

$$
\|f(t, z)-f(t, y)\| \leq L\|z-y\|,
$$

for all $z, y \in B$ and $t \geq 0$.

The next notion of the $\epsilon$-regular map is due to Arrieta and Carvalho [2, Definition 2].

Definition 2.10 ( $\varepsilon$-regular function). For $\varepsilon>0$, we say that a map $g$ is $\varepsilon$-regular relative to the pair $\left(X^{1}, X\right)$ if there exists $\rho>1, \gamma(\varepsilon)$ with $\rho \varepsilon \leq \gamma(\varepsilon)<1$ and $c>0$ such that $g: X^{1+\varepsilon} \rightarrow X^{\gamma(\varepsilon)}$ satisfying

$$
\begin{gathered}
\|g(x)-g(y)\|_{X^{\gamma(\varepsilon)}} \leq c\left(1+\|x\|_{X^{1+\varepsilon}}^{\rho-1}+\|y\|_{X^{1+\varepsilon}}^{\rho-1}\right)\|x-y\|_{X^{1+\varepsilon}}, \\
x, y \in X^{1+\varepsilon} .
\end{gathered}
$$

3. Locally and globally mild solutions. In this section, we show our main results concerning the existence of locally and globally mild solutions to the Volterra equation (2.1). We will need the following set of hypotheses:

$(\mathrm{H})_{A} A$ is the generator of an integral resolvent $(S(t))_{t>0}$;

$(\mathrm{H})_{a}$ There exist $a, b \in L_{\text {loc }}^{1}\left(\mathbb{R}_{+}\right)$satisfying the condition

$$
(a * b)(t)=1 \text { for all } t>0 .
$$

We observe that Gripenberg [11] studied assumptions on $a(t)$ in order to verify the hypothesis $(H)_{a}$. This kind of hypothesis for a related problem was recently used in [17].

Example 3.1. Let $a(t)=g_{\alpha}(t)$ where $0<\alpha<1$. Then, $b(t)=g_{1-\alpha}(t)$ satisfies $a * b(t)=g_{\alpha} * g_{1-\alpha}(t)=g_{1}(t)=1$.

The main results in this section are the following three theorems. 
Theorem 3.2 (Existence of a local solution). Assume that $(\mathrm{H})_{A}$ and $(\mathrm{H})_{a}$ hold. Let

$$
f:[0, \infty) \times X \longrightarrow X
$$

be a continuous function, uniformly and locally Lipschitz with respect to the first and second variables, respectively. Then, there exists a $t_{0}>0$ such that (2.1) has a unique local mild solution in $\left[0, t_{0}\right]$.

Proof. Given $u_{0} \in X$, let $B\left(u_{0} ; r\right)$ be the open ball with center $u_{0}$ and radius $r>0$, and let $L=L\left(B\left(u_{0} ; r\right)\right)$ be the Lipschitz constant of $f$ associated with $B\left(u_{0} ; r\right)$. Fix $\beta \in(0, r)$. By the assumptions and Remark 2.4, there exists a resolvent family $(R(t))_{t \geq 0}$ generated by $A$. Since the family $(R(t))_{t \geq 0}$ is strongly continuous and $f$ is continuous, we can choose $t_{1}$ such that

$$
\left\|R(t) u_{0}-u_{0}\right\|<\frac{\beta}{2}, \quad t \in\left[0, t_{1}\right]
$$

and

$$
C:=C_{t_{1}}:=\sup _{s \in\left[0, t_{1}\right]}\left\|f\left(s, u_{0}\right)\right\| .
$$

Now, since $a \in L_{\mathrm{loc}}^{1}\left(\mathbb{R}_{+}\right)$, we have that

$$
\lim _{t \rightarrow 0} \int_{0}^{t}|a(s)| d s=0 .
$$

Thus, we can choose $t_{0}<t_{1}$ such that

$$
\int_{0}^{t_{0}}|a(s)| d s<\frac{\beta}{2(L M \beta+M C)},
$$

where $M=M\left(t_{0}\right)$ is the bound in (2.6) and (2.7), see Remark 2.6. Let $K:=\left\{u \in C\left(\left[0, t_{0}\right] ; X\right): u(0)=u_{0},\left\|u(t)-u_{0}\right\| \leq \beta\right.$ for all $\left.t \in\left[0, t_{0}\right]\right\}$, with norm

$$
\|u\|_{K}:=\sup _{s \in\left[0, t_{0}\right]}\|u(s)\|
$$

We define a map

$$
T: K \longrightarrow K
$$

by

$$
T u(t)=R(t) u_{0}+\int_{0}^{t} S(t-s) f(s, u(s)) d s .
$$


We prove that $T(K) \subset K$. Indeed, first we show that $T u \in$ $C\left(\left[0, t_{0}\right] ; X\right)$. Let $t, t+h \in\left[0, t_{0}\right]$ with $h>0$ be given. Then,

$$
\begin{aligned}
& \|T u(t+h)-T u(t)\| \\
& \leq\left\|R(t+h) u_{0}-R(t) u_{0}\right\| \\
& \quad+\left\|\int_{0}^{t+h} S(t+h-s) f(s, u(s)) d s-\int_{0}^{t} S(t-s) f(s, u(s)) d s\right\| \\
& \leq\left\|R(t+h) u_{0}-R(t) u_{0}\right\|+\int_{0}^{t}\|[S(t+h-s)-S(t-s)] f(s, u(s)) d s\| \\
& \quad+\int_{t}^{t+h}\|S(t+h-s) f(s, u(s)) d s\| \\
& =: I_{1}+I_{2}+I_{3} .
\end{aligned}
$$

By the strong continuity of $(R(t))_{t \geq 0}$, we have $I_{1} \rightarrow 0$ as $h \rightarrow 0$. We claim that $I_{2}, I_{3} \rightarrow 0$ as $h \rightarrow 0$. In fact, since $(S(t))_{t>0}$ is strongly continuous on $X$, we have that

$$
\|S(t+h-s) f(s, u(s))-S(t-s) f(s, u(s))\| \longrightarrow 0
$$

as $h \rightarrow 0$. Also, $\|S(t-s) f(s, u(s))\|$ is dominated by $M C|a(t-s)|$, which is an integrable function. It follows from the dominated convergence theorem that $I_{2} \rightarrow 0$ as $h \rightarrow 0$. Now, since $a \in L_{\text {loc }}^{1}\left(\mathbb{R}_{+}\right)$, we obtain

$$
\begin{aligned}
I_{3} & =\int_{t}^{t+h}\|S(t+h-s) f(s, u(s))\| d s \\
& \leq M C \int_{t}^{t+h}|a(t+h-s)| d s \\
& =M C \int_{0}^{h} a(\tau) d \tau \longrightarrow 0, \quad h \rightarrow 0 .
\end{aligned}
$$

This proves the claim and shows that $T u \in C\left(\left[0, t_{0}\right] ; X\right)$. Second, note that $T u(0)=u_{0}$ since $R(0)=I$ and, for $t \in\left[0, t_{0}\right]$,

$$
\begin{aligned}
\left\|\int_{0}^{t} S(t-s) f(s, u(s)) d s\right\| \leq & \int_{0}^{t}\|S(t-s)\|\left\|f(s, u(s))-f\left(s, u_{0}\right)\right\| d s \\
& +\int_{0}^{t}\|S(t-s)\|\left\|f\left(s, u_{0}\right)\right\| d s \\
\leq & M L \int_{0}^{t} \mid a(t-s)\|\| u(s)-u_{0} \| d s
\end{aligned}
$$




$$
\begin{aligned}
& +M \int_{0}^{t}|a(t-s)|\left\|f\left(s, u_{0}\right)\right\| d s \\
\leq & (M L \beta+M C) \int_{0}^{t}|a(s)| d s \longrightarrow 0, \text { as } t \rightarrow 0 .
\end{aligned}
$$

Hence, it remains to show that $\left\|T u(t)-u_{0}\right\| \leq \beta$ for all $t \in\left[0, t_{0}\right]$. Indeed, for $t \in\left[0, t_{0}\right]$, we obtain

$$
\begin{aligned}
\left\|T u(t)-u_{0}\right\| \leq & \left\|R(t) u_{0}-u_{0}\right\|+\int_{0}^{t}\|S(t-s)\|\|f(s, u(s))\| d s \\
\leq & \left\|R(t) u_{0}-u_{0}\right\|+\int_{0}^{t}\|S(t-s)\|\left\|f(s, u(s))-f\left(s, u_{0}\right)\right\| d s \\
& +\int_{0}^{t}\|S(t-s)\|\left\|f\left(s, u_{0}\right)\right\| d s \\
\leq & \left\|R(t) u_{0}-u_{0}\right\|+M L \int_{0}^{t}|a(t-s)|\left\|u(s)-u_{0}\right\| d s \\
& +M \int_{0}^{t}|a(t-s)|\left\|f\left(s, u_{0}\right)\right\| d s \\
\leq & \left\|R(t) u_{0}-u_{0}\right\|+(M L \beta+M C) \int_{0}^{t_{0}}|a(s)| d s<\beta,
\end{aligned}
$$

where we have used the estimates (3.1) and (3.2). This shows the claim and proves that $T(K) \subset K$.

Next, we prove that $T$ is a contraction. Indeed,

$$
\begin{aligned}
\|T u(t)-T v(t)\| & \leq \int_{0}^{t}\|S(t-s)\|\|f(s, u(s))-f(s, v(s))\| d s \\
& \leq M L \int_{0}^{t}|a(t-s)\|\mid u(s)-v(s)\| d s \\
& \leq M L\|u-v\|_{K} \int_{0}^{t_{0}}|a(s)| d s \\
& <\frac{1}{2}\|u-v\|_{K},
\end{aligned}
$$

where we used the estimate (3.2). By the Banach fixed point theorem, we obtain that equation (2.1) has a unique locally mild solution in $\left[0, t_{0}\right]$. 
We next recall the following notions.

Definition 3.3. Let $u:[0, \tau] \rightarrow X$ be the unique locally mild solution in $[0, \tau]$ to $(2.1)$. If $\tau^{*}>\tau$ and

$$
u:\left[0, \tau^{*}\right] \longrightarrow X
$$

is a locally mild solution to $(2.1)$, then we say that $u^{*}$ is a continuation of $u$ over $\left[0, \tau^{*}\right]$.

Definition 3.4. Let $u:\left[0, \tau^{*}\right) \rightarrow X$ be the unique local mild solution in $[0, \tau]$ to $(2.1)$ for all $\tau \in\left(0, \tau^{*}\right)$ that does not have a continuation. Then we call it a maximal local mild solution.

Our second main result in this section is the next theorem regarding continuation of mild solutions.

Theorem 3.5 (Extension). Assume that $(\mathrm{H})_{A}$ and $(\mathrm{H})_{a}$ hold. Let

$$
f:[0, \infty) \times X \longrightarrow X
$$

be a continuous function, uniformly and locally Lipschitz with respect to the first and second variable, respectively. If $u:\left[0, t_{0}\right] \rightarrow X$ is the unique locally mild solution to $(2.1)$ in $\left[0, t_{0}\right]$, then there exists a unique continuation $u^{*}$ of $u$ in some interval $\left[0, t_{0}+\tau\right]$ with $\tau>0$.

Proof. Let

$$
u:\left[0, t_{0}\right] \longrightarrow X
$$

be the unique mild solution of $(2.1)$ in $\left[0, t_{0}\right]$. Since $f$ is locally Lipschitz, there exist $r>0$, an open ball $B:=B\left(u\left(t_{0}\right), r\right)$ and the Lipschitz constant $L>0$ of $f$ depending upon $B$. Fix $\beta \in(0, r)$. By the strong continuity of $(R(t))_{t \geq 0}$, there exists a $t_{1}>t_{0}$ such that

$$
\left\|R(t) u_{0}-R\left(t_{0}\right) u_{0}\right\|<\frac{\beta}{3}, \quad t \in\left[t_{0}, t_{1}\right] .
$$

The dominated convergence theorem shows that

$$
\int_{0}^{t_{0}}\left\|S(t-s) f(s, u(s))-S\left(t_{0}-s\right) f(s, u(s))\right\| d s \longrightarrow 0, \quad t \rightarrow t_{0} .
$$


Thus, we choose $t_{2}>t_{0}$ such that

$$
\begin{gathered}
\int_{0}^{t_{0}}\left\|S(t-s) f(s, u(s))-S\left(t_{0}-s\right) f(s, u(s))\right\| d s<\frac{\beta}{3} \\
t \in\left[t_{0}, t_{2}\right] .
\end{gathered}
$$

Let $t_{3}=\min \left\{t_{1}, t_{2}\right\}$ and $C:=C_{t_{3}}:=\sup _{s \in\left[t_{0}, t_{0}+t_{3}\right]}\left\|f\left(s, u\left(t_{0}\right)\right)\right\|$. Note that

$$
\lim _{t \rightarrow t_{0}^{+}} \int_{0}^{t-t_{0}}|a(s)| d s=0 .
$$

Then, we can take $t_{0}<t_{0}+\tau<t_{3}$ such that

$$
\int_{0}^{t-t_{0}}|a(s)| d s<\frac{\beta}{3(L M \beta+M C)}, \quad t_{0} \leq t \leq t_{0}+\tau,
$$

where $M=M\left(t_{0}\right)$ is the bound in the estimates (2.6) and (2.7).

Let

$$
\begin{aligned}
K:=\left\{w \in C\left(\left[0, t_{0}+\tau\right] ; X\right): w(t)=u(t)\right. & \text { for all } t \in\left[0, t_{0}\right], \\
\left\|w(t)-u\left(t_{0}\right)\right\| \leq \beta & \text { for all } \left.t \in\left[t_{0}, t_{0}+\tau\right]\right\}
\end{aligned}
$$

with norm

$$
\|w\|_{K}:=\sup _{s \in\left[0, t_{0}+\tau\right]}\|w(s)\|
$$

We define the map

$$
T: K \longrightarrow K
$$

by

$$
T w(t)=R(t) u_{0}+\int_{0}^{t} S(t-s) f(s, w(s)) d s .
$$

Next, we prove that $T(K) \subset K$. As in the proof of Theorem 3.2, we can prove that $T w \in C\left(\left[0, t_{0}+\tau\right] ; X\right)$ and

$$
\begin{aligned}
T w(t) & =R(t) u_{0}+\int_{0}^{t} S(t-s) f(s, w(s)) d s \\
& =R(t) u_{0}+\int_{0}^{t} S(t-s) f(s, u(s)) d s=u(t), \quad t \in\left[0, t_{0}\right] .
\end{aligned}
$$


It remains to show that

$$
\left\|T w(t)-u\left(t_{0}\right)\right\| \leq \beta \quad \text { for all } t \in\left[t_{0}, t_{0}+\tau\right] .
$$

Indeed, for $t \in\left[t_{0}, t_{0}+\tau\right]$, we have

$$
\begin{aligned}
\left\|T w(t)-u\left(t_{0}\right)\right\| \leq & \left\|R(t) u_{0}-R\left(t_{0}\right) u_{0}\right\| \\
& +\int_{0}^{t_{0}}\left\|S(t-s) f(s, u(s))-S\left(t_{0}-s\right) f(s, u(s))\right\| d s \\
& +\int_{t_{0}}^{t}\|S(t-s) f(s, w(s))\| d s,
\end{aligned}
$$

where we have used that $u\left(t_{0}\right)=T u\left(t_{0}\right)=T w\left(t_{0}\right)$. Now, from (2.6) and (2.7), together with the local Lipschitz condition, we deduce the following inequalities:

$$
\begin{aligned}
\int_{t_{0}}^{t} & \|S(t-s) f(s, u(s))\| d s \\
\leq & M \int_{t_{0}}^{t}|a(t-s)|\left\|f(s, w(s))-f\left(s, u\left(t_{0}\right)\right)\right\| d s \\
& +M \int_{t_{0}}^{t}|a(t-s)|\left\|f\left(s, u\left(t_{0}\right)\right)\right\| d s \\
\leq & M L \int_{t_{0}}^{t}|a(t-s)|\left\|w(s)-u\left(t_{0}\right)\right\| d s \\
& +M C \int_{t_{0}}^{t}|a(t-s)| d s \\
\leq & (M L \beta+M C) \int_{0}^{t-t_{0}}|a(s)| d s<\frac{\beta}{3},
\end{aligned}
$$

where we have used that $t_{0} \leq t \leq t_{0}+\tau<t_{3}, w \in K$, and the inequality (3.5). It follows from (3.3), (3.4) and the previous inequality that $\left\|T w(t)-u\left(t_{0}\right)\right\|<\beta$. Hence, $T(K) \subset K$.

Finally, observe that, for $t \in\left[0, t_{0}\right]$ and $w, v \in K$, we have that $\|(T w)(t)-(T v)(t)\|=\|u(t)-u(t)\|=0$. If $t \in\left[t_{0}, t_{0}+\tau\right]$, we obtain

$$
\|(T w)(t)-(T v)(t)\| \leq \int_{t_{0}}^{t}\|S(t-s)\|\|f(s, w(s))-f(s, v(s))\| d s
$$




$$
\begin{aligned}
& \leq M L \int_{t_{0}}^{t} a(t-s)\|w(s)-v(s)\| d s \\
& \leq M L\|w-v\|_{K} \int_{0}^{t-t_{0}} a(s) d s \\
& <\frac{1}{3}\|u-v\|_{K}, \quad w, v \in K .
\end{aligned}
$$

Hence, equation (2.1) has a unique, locally mild solution in $\left[0, t_{0}+\tau\right]$.

The next lemma provides a technical tool needed for the proof of the forthcoming main theorem.

Lemma 3.6. Assume that $(\mathrm{H})_{A}$ and $(\mathrm{H})_{a}$ hold. Let $\omega \in(0, \infty)$, and let

$$
u:[0, \infty) \longrightarrow X
$$

be a bounded continuous function. Suppose that

$$
f:[0, \infty) \times X \longrightarrow X
$$

is a continuous function that maps bounded sets onto bounded sets. If $\left(t_{n}\right) \subset[0, \omega)$ satisfies $\lim _{n \rightarrow \infty} t_{n}=\omega$, then

$$
\lim _{n \rightarrow \infty} \int_{0}^{t_{n}}\left\|\left[S\left(t_{n}-r\right)-S(\omega-r)\right] f(r, u(r))\right\| d r=0 .
$$

Proof. By hypothesis, we have that $K_{0}:=\sup _{s \in[0, \omega]}\|f(s, u(s))\|<$ $\infty$. Let $\varepsilon>0$ be given. We can take $0<h<\omega$ such that

$$
\int_{0}^{\omega-h}|a(s)| d s<\frac{\varepsilon}{4 M K_{0}} .
$$

On the other hand, since $\left(t_{n}\right) \subset[0, \omega)$ satisfies $\lim _{n \rightarrow \infty} t_{n}=\omega$, there exists an $N_{1} \in \mathbb{N}$ large enough such that $\left(t_{n}\right) \subset(h, \omega)$ for $n>N_{1}$. In this case, we have

$$
\begin{aligned}
& \left\|\left[R\left(t_{n}-s\right)-R(\omega-s)\right] f(s, u(s))\right\| \leq M K_{0}\left(a\left(t_{n}-s\right)-a(\omega-s)\right) \leq M_{N_{1}, \omega}, \\
& 0<s<h \text { and } n>N_{1} \text {, since } a \in C((0, \infty)) \text { and } \omega>t_{n}>h \text {. Then, } \\
& \text { by the strong continuity of }(S(t))_{t>0} \text { and the dominated convergence }
\end{aligned}
$$


theorem, we can take $N>N_{1}$ large enough such that

$$
\int_{0}^{h}\left\|\left[S\left(t_{n}-s\right)-S(\omega-s)\right] f(s, u(s))\right\| d s<\frac{\varepsilon}{2}, \quad n>N .
$$

Therefore, for $n>N$, we obtain

$$
\begin{aligned}
\int_{0}^{t_{n}} & \left\|\left[S\left(t_{n}-s\right)-S(\omega-s)\right] f(s, u(s))\right\| d s \\
\leq & \int_{0}^{h}\left\|\left[S\left(t_{n}-s\right)-S(\omega-s)\right] f(s, u(s))\right\| d s \\
& +\int_{h}^{t_{n}}\left\|\left[S\left(t_{n}-s\right)-S(\omega-s)\right] f(s, u(s))\right\| d s \\
& <\frac{\varepsilon}{2}+M K_{0} \int_{h}^{t_{n}}\left(\left|a\left(t_{n}-s\right)\right|+|a(\omega-s)|\right) d s \\
\leq & \frac{\varepsilon}{2}+M K_{0}\left(\int_{0}^{t_{n}-h}|a(u)| d u+\int_{0}^{w-h}|a(u)| d u\right) \\
\leq & \frac{\varepsilon}{2}+2 M K_{0} \int_{0}^{\omega-h}|a(u)| d u<\varepsilon .
\end{aligned}
$$

The result is concluded.

The third main result in this section is the following theorem.

Theorem 3.7 (Blow up). Assume that $(\mathrm{H})_{A}$ and $(\mathrm{H})_{a}$ hold. Let

$$
f:[0, \infty) \times X \longrightarrow X
$$

be a bounded continuous function, uniformly and locally Lipschitz with respect to the first and second variables, respectively. Then, either equation (2.1) has a globally mild solution in $[0, \infty)$ or there exists an $\omega \in(0, \infty)$ such that

$$
u:[0, \omega) \longrightarrow X
$$

is a maximal locally mild solution, and, in this case, $\lim _{t \rightarrow \omega^{-}}\|u(t)\|=$ $\infty$. 
Proof. We define the set

$$
H:=\left\{\tau \in[0, \infty): \text { there exists a } u_{\tau}:[0, \tau] \longrightarrow X\right.
$$

unique mild solution to $(2.1)$ on $[0, \tau]\}$.

We denote $\omega=\sup _{\tau \in H} \tau$, and consider a continuous function $u$ : $[0, \omega) \rightarrow X$ which is a locally mild solution to equation $(2.1)$ in $[0, \omega)$. If $\omega=\infty$, then $u$ is a globally mild solution in $[0, \infty)$. Otherwise, if $\omega<\infty$, then we will prove that

$$
\limsup _{t \rightarrow \omega^{-}}\|u(t)\|=\infty
$$

Indeed, suppose that this is not true. Then, there exists a $K_{0}>0 \mathrm{such}$ that

$$
\|u(t)\| \leq K_{0} \quad \text { for all } t \in[0, \omega)
$$

Since $f$ is bounded,

$$
K:=\sup _{t \in[0, \omega)}\|f(t, u(t))\|<\infty .
$$

On the other hand, it follows from the strong continuity of $(R(t))_{t \geq 0}$ and Lemma 3.6 that, if $\left(t_{n}\right) \subset[0, \omega)$ is a sequence that converges to $\omega$, then, given $\varepsilon>0$, there exists an $N \in \mathbb{N}$ such that, for all $m, n \geq N$, we have

$$
\begin{gathered}
\left\|R\left(t_{n}\right) u_{0}-R\left(t_{m}\right) u_{0}\right\|<\frac{\varepsilon}{4}, \\
\int_{0}^{t_{n}}\left\|\left[S\left(t_{n}-r\right) f(r, u(r))-S(\omega-r) f(r, u(r))\right]\right\| d r<\frac{\varepsilon}{4}, \\
\int_{0}^{t_{m}}\left\|\left[S(\omega-r) f(r, u(r))-S\left(t_{m}-r\right) f(r, u(r))\right]\right\| d r<\frac{\varepsilon}{4},
\end{gathered}
$$

and

$$
M K \int_{0}^{\omega-t_{m}}|a(s)| d s<\frac{\varepsilon}{4} .
$$

Hence, for $n, m \geq N$ and assuming, without loss of generality, that $t_{n}>t_{m}$, we obtain 


$$
\begin{aligned}
&\left\|u\left(t_{n}\right)-u\left(t_{m}\right)\right\| \leq\left\|R\left(t_{n}\right) u_{0}-R\left(t_{m}\right) u_{0}\right\| \\
&+\left\|\int_{0}^{t_{n}} S\left(t_{n}-r\right) f(r, u(r)) d r-\int_{0}^{t_{m}} S\left(t_{m}-r\right) f(r, u(r)) d r\right\| \\
& \leq\left\|R\left(t_{n}\right) u_{0}-R\left(t_{m}\right) u_{0}\right\| \\
& \quad+\int_{0}^{t_{n}}\left\|\left[S\left(t_{n}-r\right) f(r, u(r))-S(\omega-r) f(r, u(r))\right]\right\| d r \\
& \quad+\int_{0}^{t_{m}}\left\|\left[S(\omega-r) f(r, u(r))-S\left(t_{m}-r\right) f(r, u(r))\right]\right\| d r \\
& \quad+\int_{t_{m}}^{t_{n}}\|S(\omega-r) f(r, u(r))\| d r \\
& \leq\left\|R\left(t_{n}\right) u_{0}-R\left(t_{m}\right) u_{0}\right\| \\
&+\int_{0}^{t_{n}}\left\|\left[S\left(t_{n}-r\right) f(r, u(r))-S(\omega-r) f(r, u(r))\right]\right\| d r \\
&+\int_{0}^{t_{m}}\left\|\left[S(\omega-r) f(r, u(r))-S\left(t_{m}-r\right) f(r, u(r))\right]\right\| d r \\
&+M K \int_{0}^{\omega-t_{m}}|a(r)| d r<\varepsilon,
\end{aligned}
$$

where we used (2.6) in the last integral. This implies that $\left\{u\left(t_{n}\right)\right\}$ is a Cauchy sequence in $X$. Hence, there exists

$$
u(\omega):=\lim _{n \rightarrow \infty} u\left(t_{n}\right) .
$$

Now, observe that

$$
\begin{aligned}
& \left\|\int_{0}^{t_{n}} S\left(t_{n}-r\right) f(r, u(r)) d r-\int_{0}^{\omega} S(\omega-r) f(r, u(r)) d r\right\| \\
& \leq \int_{0}^{t_{n}}\left\|\left[S\left(t_{n}-r\right) f(r, u(r))-S(\omega-r) f(r, u(r))\right]\right\| d r \\
& \quad+\int_{t_{n}}^{\omega}\|S(\omega-r) f(r, u(r))\| d r \\
& \leq \int_{0}^{t_{n}}\left\|\left[S\left(t_{n}-r\right) f(r, u(r))-S(\omega-r) f(r, u(r))\right]\right\| d r \\
& \quad+M K \int_{0}^{\omega-t_{n}}|a(r)| d r \longrightarrow 0,
\end{aligned}
$$


as $n \rightarrow \infty$. It follows that

$$
\begin{aligned}
u(\omega): & =\lim _{n \rightarrow \infty}\left[R\left(t_{n}\right) u_{0}+\int_{0}^{t_{n}} S\left(t_{n}-s\right) f(s, w(s)) d s\right] \\
& =R(\omega) u_{0}+\int_{0}^{\omega} S(\omega-s) f(s, w(s)) d s .
\end{aligned}
$$

Hence, we can extend $u$ over $[0, \omega]$. Then, by the extension Theorem 3.5, we can extend the solution to some bigger interval, a contradiction by the definition of $\omega$. Therefore, $\omega<\infty$ implies that $\lim _{t \rightarrow \omega^{-}}\|u(t)\|=\infty$.

4. Spatial regularity and $\varepsilon$-mild regular solutions for $a(t)=$ $\left(t^{\alpha-1} / \Gamma(\alpha)\right) e^{-\delta t}$. Let $0<\alpha<1$ and $\delta \geq 0$ be given. In this section, we need the condition:

(H) $-A$ is a sectorial operator of angle $\theta \in[0, \pi / 2)$ and

$$
a(t):=\frac{t^{\alpha-1}}{\Gamma(\alpha)} e^{-\delta t} .
$$

We note that $a(t)$ defined as above is the usual kernel employed in linear viscoelastic theory for the analysis of Volterra equations of convolution type, see [23, subsection 5.2].

In the case of $\delta=0$, it is well known that, under condition $(\mathrm{H})$, there exist strongly continuous families of bounded and linear operators $\left(S_{\alpha}(t)\right)_{t>0}$ and $\left(R_{\alpha}(t)\right)_{t>0}$, defined by

$$
S_{\alpha}(t):=\frac{1}{2 \pi i} \int_{\gamma} e^{\lambda t}\left(\lambda^{\alpha}-A\right)^{-1} d \lambda, \quad t>0
$$

and

$$
R_{\alpha}(t):=\frac{1}{2 \pi i} \int_{\gamma} e^{\lambda t} \lambda^{\alpha-1}\left(\lambda^{\alpha}-A\right)^{-1} d \lambda, \quad t>0,
$$

where $\gamma \subset \rho(A)$ is a Hankel's path. We recall that a complex path $\gamma$ is called a Hankel's path if there exist $r>0$ and $\omega \in(0, \pi)$ such that

$$
\gamma=\gamma_{r, \omega}:=\left\{\lambda e^{i \omega}: \lambda \geq r\right\} \cup\left\{r e^{i \varphi}: \varphi \in(-\omega, \omega)\right\} \cup\left\{\lambda e^{-i \omega}: \lambda \geq r\right\},
$$

oriented counterclockwise. The family $(R(t))_{t>0}$ can be extended in $t=0$ to the identity operator by strong continuity. It is known that the 
families $\left(S_{\alpha}(t)\right)_{t>0}$ and $\left(R_{\alpha}(t)\right)_{t \geq 0}$ correspond to an integral resolvent and resolvent family, respectively, generated by $A$ (see [8, Lemma 2.1]).

Remark 4.1. If $A$ is bounded, then

$$
S_{\alpha}(t)=t^{\alpha-1} E_{\alpha, \alpha}\left(t^{\alpha} A\right):=t^{\alpha-1} \sum_{n=0}^{\infty} \frac{\left(t^{\alpha} A\right)^{n}}{\Gamma(\alpha n+\alpha)},
$$

and

$$
R_{\alpha}(t)=E_{\alpha}\left(t^{\alpha} A\right):=\sum_{n=0}^{\infty} \frac{\left(t^{\alpha} A\right)^{n}}{\Gamma(\alpha n+1)},
$$

where $E_{\alpha, \beta}$ denotes the generalized Mittag-Leffler function, see e.g., [16]).

Remark 4.2. Let $0<\alpha<1$ and $0 \leq \beta \leq 1$ be given. It is known that the following spatial regularity holds:

(i) $\left\|S_{\alpha}(t) x\right\| \leq M t^{\alpha-1}\|x\|, t>0, x \in X$;

(ii) $\left\|S_{\alpha}(t) x\right\|_{X^{\beta}} \leq M t^{\alpha(1-\beta)-1}\|x\|, t>0, x \in X$;

(iii) $\left\|R_{\alpha}(t) x\right\| \leq M\|x\|, t \geq 0, x \in X$;

(iv) $\left\|R_{\alpha}(t) x\right\|_{X^{\beta}} \leq M t^{-\alpha \beta}\|x\|, t>0, x \in X$.

For a proof and more information on these properties, see e.g., [8, Lemma 2.2] and [13, Theorems 2.1, 2.2].

Concerning the case $\delta \neq 0$, we construct the following operator families.

Lemma 4.3. Set $0<\alpha<1, \delta \geq 0$, and assume that $(\mathrm{H})$ holds. Then, A generates the integral resolvent

$$
S_{\alpha, \delta}(t):=e^{-\delta t} S_{\alpha}(t), \quad t>0,
$$

and the resolvent family

$$
R_{\alpha, \delta}(t):=e^{-\delta t} R_{\alpha}(t)+\delta \int_{0}^{t} e^{-\delta s} R_{\alpha}(s) d s, \quad t \geq 0 .
$$


Proof. The first part follows from the identity

$$
\begin{aligned}
e^{-\delta t} S_{\alpha}(t) x= & e^{-\delta t} g_{\alpha}(t) x \\
& +\int_{0}^{t} e^{-\delta(t-s)} g_{\alpha}(t-s) A e^{-\delta s} S_{\alpha}(s) x d s, \quad t>0 .
\end{aligned}
$$

The second claim follows from (2.4) observing that, for $a(t)=$ $g_{\alpha}(t) e^{-\delta t}$,

$$
b(t)=g_{1-\alpha}(t) e^{-\delta t}+\delta \int_{0}^{t} g_{1-\alpha}(s) e^{-\delta s} d s
$$

satisfies condition $(\mathrm{H})_{a}$.

For each $0<\alpha<1, \beta>0$ and $\delta \geq 0$, we define the functions

$$
\psi_{\alpha, \beta, \delta}(t):=t^{\alpha(1-\beta)-1} e^{-\delta t}, \quad t>0,
$$

and

$$
\varphi_{\alpha, \beta, \delta}(t):=e^{-\delta t} t^{-\alpha \beta}+\delta \int_{0}^{t} e^{-\delta s} s^{-\alpha \beta} d s, \quad t>0 .
$$

The relation between both of the above functions is given as follows.

Lemma 4.4. For each $0<\alpha<1, \beta>0$ and $\delta \geq 0$, we have

$$
\varphi_{\alpha, \beta, \delta}=\frac{\Gamma(1-\alpha \beta)}{\Gamma(\alpha(1-\beta))}\left(b * \psi_{\alpha, \beta, \delta}\right) .
$$

Proof. First, note that

$$
\left(e^{-\delta \cdot} g_{1-\alpha}\right) *\left(e^{-\delta \cdot} g_{\alpha(1-\beta)}\right)=e^{-\delta \cdot} g_{1-\alpha \beta} .
$$

Then,

$$
\begin{aligned}
\frac{1}{\Gamma(\alpha(1-\beta))}\left(b * \psi_{\alpha, \beta, \delta}\right)(t) & =e^{-\delta t} g_{1-\alpha \beta}+\delta\left(g_{1} *\left(e^{-\delta \cdot} g_{1-\alpha \beta}\right)\right)(t) \\
& =\frac{1}{\Gamma(1-\alpha \beta)} \varphi_{\alpha, \beta, \delta}(t)
\end{aligned}
$$

One of the main results in this section is the next theorem concerning the spatial regularity of the families $\left(S_{\alpha, \delta}(t)\right)_{t>0}$ and $\left(R_{\alpha, \delta}(t)\right)_{t \geq 0}$. 
Theorem 4.5. Let $0<\alpha<1$ and $\delta \geq 0$ be given, and assume that $(\mathrm{H})$ is satisfied. There exists a constant $M>0$ such that the following properties hold.

(a) (i) $\left\|S_{\alpha, \delta}(t) x\right\| \leq M e^{-\delta t} t^{\alpha-1}\|x\|, t>0, x \in X$.

(ii) $\left\|R_{\alpha, \delta}(t) x\right\| \leq M\|x\|, t \geq 0, x \in X$.

(b) For any $0 \leq \beta \leq 1$, we have

(i) $\left\|S_{\alpha, \delta}(t) x\right\|_{X^{\beta}} \leq M \psi_{\alpha, \beta, \delta}(t)\|x\|, x \in X$.

(ii) $\left\|R_{\alpha, \delta}(t) x\right\|_{X^{\beta}} \leq M \varphi_{\alpha, \beta, \delta}(t)\|x\|, x \in X$.

(c) For any $0<\beta<1$, we have

(i) $\left\|S_{\alpha, \delta}(t) x\right\|_{X^{1}} \leq M \psi_{\alpha, 1-\beta, \delta}(t)\|x\|_{X^{\beta}}, x \in X^{\beta}$.

(ii) $\left\|R_{\alpha, \delta}(t) x\right\|_{X^{1}} \leq M \varphi_{\alpha, 1-\beta, \delta}(t)\|x\|_{X^{\beta}}, x \in X^{\beta}$.

(d) For any $0 \leq \theta \leq \beta \leq 1$, we have

(i) $\left\|S_{\alpha, \delta}(t) x\right\|_{X^{1+\theta}} \leq M \psi_{\alpha, 1+\theta-\beta, \delta}(t)\|x\|_{X^{\beta}}, x \in X^{\beta}$.

(ii) $\left\|R_{\alpha, \delta}(t) x\right\|_{X^{1+\theta}} \leq M \varphi_{\alpha, 1+\theta-\beta, \delta}(t)\|x\|_{X^{\beta}}, x \in X^{\beta}$.

Proof. Parts (a)(i) and (ii) directly follows from Remark 4.2 (i) and (iii), respectively. Part (b) follows from Remark 4.2 (ii) and (iv), respectively. Part (c) follows from (b) and the identities

$$
\begin{aligned}
& A S_{\alpha, \delta}(t) x=A^{1-\beta} S_{\alpha, \delta}(t) A^{\beta} x, \\
& A R_{\alpha, \delta}(t) x=A^{1-\beta} R_{\alpha, \delta}(t) A^{\beta} x,
\end{aligned}
$$

valid for all $x \in X^{\beta}$ and $0<\beta<1$. Finally, part (d) follows from the identities

$$
\begin{aligned}
& A^{1+\theta} S_{\alpha, \delta}(t) x=A^{1+\theta-\beta} S_{\alpha, \delta}(t) A^{\beta} x, \\
& A^{1+\theta} R_{\alpha, \delta}(t) x=A^{1+\theta-\beta} R_{\alpha, \delta}(t) A^{\beta} x,
\end{aligned}
$$

where $x \in X^{\beta}$.

This result allows the study of local existence of the solution for the class of Volterra equations treated in this paper with viscoelastic kernels, when the function $f$ has critical conditions. More precisely, we will study the existence of mild solutions to the equation

$$
u(t)=\int_{0}^{t} \frac{(t-s)^{\alpha-1}}{\Gamma(\alpha)} e^{-\delta(t-s)}\left[A u(s)+f(s, u(s)] d s+u_{0},\right.
$$

$t \in J:=[0, T]$, in the following sense. 
Definition 4.6. A continuous function $u:[0, T] \rightarrow X^{1}$ is called an $\varepsilon$-regular mild solution to (4.3) if $u \in C\left((0, T] ; X^{1+\varepsilon}\right)$, and it satisfies the integral equation

$$
u(t)=R_{\alpha, \delta}(t) u_{0}+\int_{0}^{t} S_{\alpha, \delta}(t-s) f(s, u(s)) d s, \quad t \in J .
$$

Definition 4.6 corresponds to a slight extension of those given in [2, Definition 1] in the case of $\delta=0$. As previously mentioned, we shall now consider a class of functions with certain critical conditions.

Definition 4.7. [2, Section 2] Let $\varepsilon, \gamma(\varepsilon), \xi, \zeta, c$ and $\delta^{\prime}$ be positive constants, and consider a real function $\nu$ with values in $\left[0, \delta^{\prime}\right)$ satisfying $\lim _{t \rightarrow 0^{+}} \nu(t)=0$. We define the $\mathcal{F}(\varepsilon, \gamma(\varepsilon), c, \nu, \xi, \zeta)$-class as the family of functions $f$ such that, for $t \geq 0, f(t, \cdot)$ is an $\varepsilon$-regular map relative to the pair $\left(X^{1}, X\right)$, satisfying for all $x, y \in X^{1+\varepsilon}$,

$$
\begin{aligned}
\|f(t, x)-f(t, y)\|_{X^{\gamma(\varepsilon)}} & \leq c\left(\|x\|_{X^{1+\varepsilon}}^{\rho-1}+\|y\|_{X^{1+\varepsilon}}^{\rho-1}+\nu(t) t^{-\zeta}\right)\|x-y\|_{X^{1+\varepsilon}} \\
\|f(t, x)\|_{X^{\gamma(\varepsilon)}} & \leq c\left(\|x\|_{X^{1+\varepsilon}}^{\rho}+\nu(t) t^{-\xi}\right) .
\end{aligned}
$$

We can assume without loss of generality that $\nu$ is non-decreasing. In addition, we will assume that $0 \leq \zeta \leq \alpha(\gamma(\varepsilon)-\varepsilon)$ and $0 \leq \xi \leq \alpha \gamma(\varepsilon)$. For convenience, we will denote the class $\mathcal{F}(\varepsilon, \gamma(\varepsilon), c, \nu, \xi, \zeta)$ by $\mathcal{F}(\nu)$.

Before stating the main result, we prove the following technical lemma.

Lemma 4.8. Let $0 \leq \theta \leq 1$ be given. We have

$$
\left\|\frac{t^{\alpha \theta}}{1+(\delta / 1-\alpha \theta) t} R_{\alpha, \delta}(t)\right\|_{\mathcal{B}\left(X^{1}, X^{1+\theta}\right)} \leq M
$$

for some constant $M>0$. Moreover, for $0<\theta \leq 1$,

$$
\lim _{t \rightarrow 0^{+}} \sup _{x \in K}\left\|\frac{t^{\alpha \theta}}{1+(\delta / 1-\alpha \theta) t} R_{\alpha, \delta}(t) x\right\|_{X^{1+\theta}}=0,
$$

where $K$ denotes a compact subset of $D(A)$. 
Proof. First, note that the bound

$$
\left\|\frac{t^{\alpha \theta}}{1+\delta /(1-\alpha \theta) t} R_{\alpha, \delta}(t)\right\|_{\mathcal{B}\left(X^{1}, X^{1+\theta}\right)} \leq M
$$

is a straightforward consequence of Theorem 4.5 (d) (ii). In order to prove the second part, we proceed as follows. Given $x \in X^{1}$, $\left\{x_{n}\right\}_{n \in \mathbb{N}} \subset X^{1+\theta}$ exists such that $x_{n} \rightarrow x$ in $X^{1}$. Thus, given $\varepsilon>0$, we take $N \in \mathbb{N}$ such that $M\left\|x_{N}-x\right\|_{X^{1}} \leq \varepsilon / 2$. Now, since $\lim _{t \rightarrow 0} t^{\alpha \theta} /[1+(\delta / 1-\alpha \theta) t]=0$ for $0<\theta \leq 1$, there exists a $0<\delta_{0}$ such that $t^{\alpha \theta} /[1+(\delta / 1-\alpha \theta) t]\left\|x_{N}\right\|_{X^{1+\theta}} \sup _{s \geq 0}\left\|R_{\alpha, \delta}(s)\right\|_{\mathcal{B}(X)}<\varepsilon / 2$ for $0<t<\delta_{0}$, where we have used Theorem 4.5 (a) (ii). Then,

$$
\begin{aligned}
\| & \frac{t^{\alpha \theta}}{1+\delta /(1-\alpha \theta) t} R_{\alpha, \delta}(t) x \|_{X^{1+\theta}} \\
\leq & \left\|\frac{t^{\alpha \theta}}{1+\delta /(1-\alpha \theta) t} R_{\alpha, \delta}(t)\left(x-x_{N}\right)\right\|_{X^{1+\theta}} \\
& +\left\|\frac{t^{\alpha \theta}}{1+\delta /(1-\alpha \theta) t} R_{\alpha, \delta}(t) x_{N}\right\|_{X^{1+\theta}} \\
\leq & M\left\|x-x_{N}\right\|_{X^{1}} \\
& +\frac{t^{\alpha \theta}}{1+\delta /(1-\alpha \theta) t}\left\|x_{N}\right\|_{X^{1+\theta}} \sup _{s \geq 0}\left\|R_{\alpha, \delta}(s)\right\|_{\mathcal{B}(X)} \\
\leq & \varepsilon / 2+\varepsilon / 2=\varepsilon,
\end{aligned}
$$

for $0<t<\delta_{0}$, where we have used Theorem 4.5 (d) (ii). Therefore,

$$
\lim _{t \rightarrow 0^{+}}\left\|\frac{t^{\alpha \theta}}{1+\delta /(1-\alpha \theta) t} R_{\alpha, \delta}(t) x\right\|_{X^{1+\theta}}=0
$$

for $x \in X^{1}$. In addition, since $K$ is compact, the result follows by standard arguments.

We will need the following result.

Lemma 4.9. Let $f \in \mathcal{F}(\nu)$ be given. If $u \in C\left((0, T] ; X^{1+\varepsilon}\right)$, then, for all $0 \leq \theta<\gamma(\varepsilon)$, we have

$$
\frac{t^{\alpha \theta}}{1+\delta /(1-\alpha \theta) t}\left\|\int_{0}^{t} S_{\alpha, \delta}(t-s) f(s, u(s)) d s\right\|_{X^{1+\theta}}
$$




$$
\leq M c \boldsymbol{B}_{\varepsilon}^{\theta}(\xi)\left(\nu(t) t^{\alpha \gamma(\varepsilon)-\xi}+\lambda(t)^{\rho} t^{\alpha(\gamma(\varepsilon)-\rho \varepsilon)} \frac{(1+\delta /(1-\alpha \varepsilon) t)^{\rho}}{1+\delta /(1-\alpha \theta) t}\right),
$$

for $0<t \leq T$, where $\lambda(t)=\sup _{s \in(0, t]} s^{\alpha \varepsilon} /[1+(\delta / 1-\alpha \varepsilon) t]\|u(s)\|_{X^{1+\varepsilon}}$ and

$$
\boldsymbol{B}_{\varepsilon}^{\theta}(\xi)=\sup _{0 \leq \eta \leq \theta}\{\boldsymbol{B}(\alpha(\gamma(\varepsilon)-\eta), 1-\xi), \boldsymbol{B}(\alpha(\gamma(\varepsilon)-\eta), 1-\alpha \rho \varepsilon)\},
$$

where $\boldsymbol{B}$ denotes the usual Beta function:

$$
\boldsymbol{B}(a, b)=\int_{0}^{1} x^{a-1}(1-x)^{b-1} d x, \quad a, b>0 .
$$

Proof. It is sufficient to observe that the following inequalities hold:

$$
\begin{aligned}
& \frac{t^{\alpha \theta}}{1+\delta /(1-\alpha \theta) t}\left\|\int_{0}^{t} S_{\alpha, \delta}(t-s) f(s, u(s)) d s\right\|_{X^{1+\theta}} \\
& \leq M c \frac{t^{\alpha \theta}}{1+\delta /(1-\alpha \theta) t} \\
& \times \int_{0}^{t}(t-s)^{\alpha(\gamma(\varepsilon)-\varepsilon)-1}\left(\nu(s) s^{-\xi}+\|u(s)\|_{X^{1+\varepsilon}}^{\rho}\right) d s \\
& \leq M c \nu(t) t^{\alpha \theta} \int_{0}^{t}(t-s)^{\alpha(\gamma(\varepsilon)-\theta)-1} s^{-\xi} d s+M c \frac{t^{\alpha \theta}}{1+\delta /(1-\alpha \theta) t} \lambda(t)^{\rho} \\
& \times \int_{0}^{t}(t-s)^{\alpha(\gamma(\varepsilon)-\theta)-1}\left(\frac{1+\delta /(1-\alpha \varepsilon) s}{s^{\alpha \varepsilon}}\right)^{\rho} d s \\
& \leq M c \nu(t) t^{\alpha \gamma(\varepsilon)-\xi} \int_{0}^{1}(1-s)^{\alpha(\gamma(\varepsilon)-\theta)-1} s^{-\xi} d s+M c t^{\alpha(\gamma(\varepsilon)-\rho \varepsilon)} \\
& \frac{(1+\delta /(1-\alpha \varepsilon) t)^{\rho}}{1+\delta /(1-\alpha \theta) t} \lambda(t)^{\rho} \\
& \times \int_{0}^{1}(1-s)^{\alpha(\gamma(\varepsilon)-\theta)-1} s^{-\alpha \rho \varepsilon} d s \\
& \leq M c \mathbf{B}_{\varepsilon}^{\theta}(\xi)\left(\nu(t) t^{\alpha \gamma(\varepsilon)-\xi}+\lambda(t)^{\rho} t^{\alpha(\gamma(\varepsilon)-\rho \varepsilon)} \frac{(1+\delta /(1-\alpha \varepsilon) t)^{\rho}}{1+\delta /(1-\alpha \theta) t}\right),
\end{aligned}
$$

where we have used Theorem $4.5(\mathrm{~d})(\mathrm{i})$ and $\left[t^{\alpha \varepsilon} /(1+\delta /(1-\alpha \varepsilon) t)\right]<$ $t^{\alpha \varepsilon}$ for $t>0$.

We next prove the following lemma. 
Lemma 4.10. Let $f \in \mathcal{F}(\nu)$ and $u, v \in C\left((0, T] ; X^{1+\varepsilon}\right)$ be such that the inequalities

$$
\frac{t^{\alpha \varepsilon}}{1+\delta /(1-\alpha \varepsilon) t}\|u(t)\|_{X^{1+\varepsilon}} \leq \mu
$$

and $t^{\alpha \varepsilon} /[1+(\delta /(1-\alpha \varepsilon) t)]\|v(t)\|_{X^{1+\varepsilon}} \leq \mu$ with $\mu>0$, holds. Then, for all $0 \leq \theta<\gamma(\varepsilon)<1$, we have the following inequality

$$
\begin{aligned}
& \frac{t^{\alpha \theta}}{1+\delta /(1-\alpha \theta) t}\left\|\int_{0}^{t} S_{\alpha, \delta}(t-s)(f(s, u(s))-f(s, v(s))) d s\right\|_{X^{1+\theta}} \\
& \leq M c \mathbf{B}_{\varepsilon}^{\theta}(\zeta+\alpha \varepsilon)\left(\nu(t) t^{\alpha(\gamma(\varepsilon)-\theta)-\zeta} \frac{1+\delta /(1-\alpha \varepsilon) t}{1+\delta /(1-\alpha \theta) t}\right. \\
& \left.\quad+2 \mu^{\rho-1} t^{\alpha(\gamma(\varepsilon)+\varepsilon-\theta-\rho \varepsilon)} \frac{(1+\delta /(1-\alpha \varepsilon) t)^{\rho}}{1+\delta /(1-\alpha \theta) t}\right) \\
& \times \sup _{0<s \leq T} \frac{s^{\alpha \varepsilon}}{1+\delta /(1-\alpha \varepsilon) s}\|u(s)-v(s)\|_{X^{1+\varepsilon}} .
\end{aligned}
$$

Proof. By the $\varepsilon$-regularity of $f$ and Theorem 4.5 (d) (i), we obtain

$$
\begin{aligned}
& \frac{t^{\alpha \theta}}{1+\delta /(1-\alpha \theta) t}\left\|\int_{0}^{t} S_{\alpha, \delta}(t-s)(f(s, u(s))-f(s, v(s))) d s\right\|_{X^{1+\theta}} \\
& \leq M c \frac{t^{\alpha \theta}}{1+\delta /(1-\alpha \theta) t} \int_{0}^{t}(t-s)^{\alpha(\gamma(\varepsilon)-\theta)-1}\|u(s)-v(s)\|_{X^{1+\varepsilon}} \\
& \times\left(\|u(s)\|_{X^{1+\varepsilon}}^{\rho-1}+\|v(s)\|_{X^{1+\varepsilon}}^{\rho-1}+\nu(s) s^{-\zeta}\right) d s \\
& \leq M c \nu(t) \frac{t^{\alpha \theta}}{1+\delta /(1-\alpha \theta) t} \\
& \times \int_{0}^{t}(t-s)^{\alpha(\gamma(\varepsilon)-\theta)-1} s^{-\zeta-\alpha \varepsilon}\left(1+\frac{\delta}{1-\alpha \varepsilon} s\right) \frac{s^{\alpha \varepsilon}}{1+\delta /(1-\alpha \varepsilon) s} \\
& \times\|u(s)-v(s)\|_{X^{1+\varepsilon}} d s+M c \frac{t^{\alpha \theta}}{1+\delta /(1-\alpha \theta) t} \\
& \times \int_{0}^{t}(t-s)^{\alpha(\gamma(\varepsilon)-\theta)-1}\left(\frac{1+\delta /(1-\alpha \varepsilon) s}{s^{\alpha \varepsilon}}\right)^{\rho} \\
& \times\left(\left(\frac{s^{\alpha \varepsilon}}{1+\delta /(1-\alpha \varepsilon) s}\|u(s)\|_{X^{1+\varepsilon}}\right)^{\rho-1}\right. \\
& \left.+\left(\frac{s^{\alpha \varepsilon}}{1+\delta /(1-\alpha \varepsilon) s}\|v(s)\|_{X^{1+\varepsilon}}\right)^{\rho-1}\right)
\end{aligned}
$$




$$
\begin{aligned}
& \times \frac{s^{\alpha \varepsilon}}{1+\delta /(1-\alpha \varepsilon) s}\|u(s)-v(s)\|_{X^{1+\varepsilon}} d s \\
& \leq M c \mathbf{B}_{\varepsilon}^{\theta}(\zeta+\alpha \varepsilon)\left(\nu(t) t^{\alpha(\gamma(\varepsilon)-\theta)-\zeta} \frac{1+\delta /(1-\alpha \varepsilon) t}{1+\delta /(1-\alpha \theta) t}\right. \\
& \left.\qquad+2 \mu^{\rho-1} t^{\alpha(\gamma(\varepsilon)+\varepsilon-\theta-\rho \varepsilon)} \frac{(1+\delta /(1-\alpha \varepsilon) t)^{\rho}}{1+\delta /(1-\alpha \theta) t}\right) \\
& \times \sup _{0<s \leq T} \frac{s^{\alpha \varepsilon}}{1+\delta /(1-\alpha \varepsilon) s}\|u(s)-v(s)\|_{X^{1+\varepsilon}},
\end{aligned}
$$

proving Lemma 4.10 .

The main result of this section is the following theorem. The proof is based on the same ideas as the proof of [8, Theorem 1.7] and uses Lemmas 4.8, 4.9, 4.10 and Theorem 4.5.

Let $B_{X^{1}}\left(v_{0}, r\right)=\left\{u \in X^{1}:\left\|u-v_{0}\right\|_{X^{1}}<r\right\}$.

Theorem 4.11. Let $f \in \mathcal{F}(\nu)$ and $v_{0} \in X^{1}$ be given. There exist $r, T_{0}>0$ such that, for any $u_{0} \in B_{X^{1}}\left(v_{0}, r\right)$, there is a continuous function

$$
u\left(\cdot, u_{0}\right):\left[0, T_{0}\right] \longrightarrow X^{1}
$$

with $u\left(0, u_{0}\right)=u_{0}$ which is an $\varepsilon$-regular mild solution to (4.3). This solution satisfies

$$
\begin{gathered}
u\left(\cdot, u_{0}\right) \in C\left(\left(0, T_{0}\right], X^{1+\theta}\right), \quad 0 \leq \theta<\gamma(\varepsilon), \\
\lim _{t \rightarrow 0^{+}} \frac{t^{\alpha \theta}}{1+\delta /(1-\alpha \theta) t}\left\|u\left(t, u_{0}\right)\right\|_{X^{1+\theta}}=0, \quad 0<\theta<\gamma(\varepsilon) .
\end{gathered}
$$

Moreover, for each $\theta_{0}<\gamma(\varepsilon)+\varepsilon-\rho \varepsilon$, there exists a $C>0$ such that, if $u_{0}, w_{0} \in B_{X^{1}}\left(v_{0}, r\right)$, then

$$
\begin{gathered}
\frac{t^{\alpha \theta}}{1+\delta /(1-\alpha \theta) t}\left\|u\left(t, u_{0}\right)-u\left(t, w_{0}\right)\right\|_{X^{1+\theta}} \leq C\left\|u_{0}-w_{0}\right\|_{X^{1}}, \\
t \in\left[0, T_{0}\right], \quad 0 \leq \theta \leq \theta_{0} .
\end{gathered}
$$

Proof. We define $\mu$ satisfying $M c \mathbf{B}_{\varepsilon}^{\varepsilon} \mu^{\rho-1}=1 / 8$, where $\mathbf{B}_{\varepsilon}^{\theta}:=$ $\max \left\{\mathbf{B}_{\varepsilon}^{\theta}(\xi), \mathbf{B}_{\varepsilon}^{\theta}(\zeta+\alpha \varepsilon)\right\}$, and we take $r=r(\mu, M)=\mu /(4 M)$. Given $v_{0}$, by Lemma 4.8 , we can choose $T_{0} \in(0,1]$ satisfying $(1+$ 
$\left.(\delta / 1-\alpha \varepsilon) T_{0}\right)^{\rho-1} \leq 2, \nu(t)<\delta^{\prime}$ and

$$
\left\|\frac{t^{\alpha \varepsilon}}{1+\delta /(1-\alpha \varepsilon) t} R_{\alpha, \delta}(t) v_{0}\right\|_{X^{1+\varepsilon}} \leq \mu / 2
$$

for $0 \leq t \leq T_{0}$, where $\delta^{\prime}$ is given by $M c \delta^{\prime} \mathbf{B}_{\varepsilon}^{\varepsilon}=\min \{\mu / 8,1 / 4\}$.

We define

$$
K\left(T_{0}\right):=\left\{u \in C\left(\left(0, T_{0}\right] ; X^{1+\varepsilon}\right): \sup _{t \in\left(0, T_{0}\right]}\left\{\frac{t^{\alpha \varepsilon}}{1+\delta /(1-\alpha \varepsilon) t}\|u(t)\|_{X^{1+\varepsilon}}\right\} \leq \mu\right\}
$$

with norm

$$
\|u\|_{K\left(T_{0}\right)}:=\sup _{t \in\left(0, T_{0}\right]} \frac{t^{\alpha \varepsilon}}{1+\delta /(1-\alpha \varepsilon) t}\|u(t)\|_{X^{1+\varepsilon}} .
$$

Let $u_{0} \in X^{1}$ be such that $\left\|u_{0}-v_{0}\right\|<r$, and define the map $T: K\left(T_{0}\right) \rightarrow K\left(T_{0}\right)$ by

$$
T u(t)=R_{\alpha, \delta}(t) u_{0}+\int_{0}^{t} S_{\alpha, \delta}(t-s) f(s, u(s)) d s .
$$

We next prove that $T\left(K\left(T_{0}\right)\right) \subset K\left(T_{0}\right)$ and $T$ is a contraction.

First, let $u \in K\left(T_{0}\right)$ be given. We shall see that $T u \in C\left(\left(0, T_{0}\right] ; X^{1+\theta}\right)$ for all $0 \leq \theta<\gamma(\varepsilon)$. In fact, let $t_{1}, t_{2} \in\left(0, T_{0}\right]$ be given. Assuming without loss of generality that $t_{1}<t_{2}$, we obtain

$$
\begin{aligned}
& \left\|T u\left(t_{2}\right)-T u\left(t_{1}\right)\right\|_{X^{1+\theta}} \\
& \leq\left\|R_{\alpha, \delta}\left(t_{2}\right) u_{0}-R_{\alpha, \delta}\left(t_{1}\right) u_{0}\right\|_{X^{1+\theta}} \\
& \quad+\left\|\int_{0}^{t_{1}}\left(S_{\alpha, \delta}\left(t_{2}-s\right)-S_{\alpha, \delta}\left(t_{1}-s\right)\right) f(s, u(s)) d s\right\|_{X^{1+\theta}} \\
& \quad+\left\|\int_{t_{1}}^{t_{2}} S_{\alpha, \delta}\left(t_{2}-s\right) f(s, u(s)) d s\right\|_{X^{1+\theta}} \cdot
\end{aligned}
$$

Using the same arguments as in [8, Claim 1, Proof of Theorem 1.7] and Theorem $4.5(\mathrm{~d})(\mathrm{i})$ we obtain that $\lim _{t_{1} \rightarrow t_{2}}\left\|T u\left(t_{2}\right)-T u\left(t_{1}\right)\right\|_{X^{1+\theta}}=0$. In addition, for $t \in\left(0, T_{0}\right]$,

$$
\begin{aligned}
& \frac{t^{\alpha \varepsilon}}{1+\delta /(1-\alpha \varepsilon) t}\|T u(t)\|_{X^{1+\varepsilon}} \\
& \quad \leq \frac{t^{\alpha \varepsilon}}{1+\delta /(1-\alpha \varepsilon) t}\left\|R_{\alpha, \delta}\left(t\left(u_{0} \mp v_{0}\right)\right)\right\|_{X^{1+\varepsilon}}+M c \frac{t^{\alpha \varepsilon}}{1+\delta /(1-\alpha \varepsilon) t}
\end{aligned}
$$




$$
\begin{aligned}
\times & \int_{0}^{t}(t-s)^{\alpha(\gamma(\varepsilon)-\varepsilon)-1}\left(\nu(s) s^{-\xi}+\|u(s)\|_{X^{1+\varepsilon}}^{\rho}\right) d s \\
\leq & M r+\frac{\mu}{2}+M c \delta^{\prime} t^{\alpha \gamma(\varepsilon)-\xi} \int_{0}^{1}(1-s)^{\alpha(\gamma(\varepsilon)-\varepsilon)-1} s^{-\xi} d s \\
& +M c \mu^{\rho} t^{\alpha(\gamma(\varepsilon)-\rho \varepsilon)} \int_{0}^{1}(1-s)^{\alpha(\gamma(\varepsilon)-\varepsilon)-1} s^{-\alpha \rho \varepsilon} d s \\
\leq & M r+\frac{\mu}{2}+M c \delta^{\prime} \mathbf{B}_{\varepsilon}^{\varepsilon}+M c \mu^{\rho} \mathbf{B}_{\varepsilon}^{\varepsilon} \leq \mu,
\end{aligned}
$$

where we used Lemma 4.8, Theorem $4.5(\mathrm{~d})(\mathrm{i})$ and $\left[t^{\alpha \varepsilon} /(1+\delta /(1-\alpha \varepsilon) t)\right]$ $<t^{\alpha \varepsilon}$ for $t>0$. Thus, we have proved that $T\left(K\left(T_{0}\right)\right) \subset K\left(T_{0}\right)$.

Second, observe that $T$ is a contraction by the choice of $T_{0}$ and Lemma 4.10 for $\theta=\varepsilon$, with

$$
\|T u-T v\|_{K\left(T_{0}\right)} \leq \frac{3}{4}\|u-v\|_{K\left(T_{0}\right)}
$$

By the Banach contraction principle, $T$ has a unique fixed point in $K\left(T_{0}\right)$. We denote that point by $u\left(\cdot, u_{0}\right)$, with $\left\|v_{0}-u_{0}\right\|_{X^{1}} \leq r$ and $0 \leq t \leq T_{0}$. Furthermore, observe that we have proved $u\left(\cdot, u_{0}\right)=$ $T u\left(\cdot, u_{0}\right) \in C\left(\left(0, T_{0}\right] ; X^{1+\theta}\right)$ for all $0 \leq \theta<\gamma(\varepsilon)$ since $u\left(\cdot, u_{0}\right) \in K\left(T_{0}\right)$. In addition, we will prove that

$$
\lim _{t \rightarrow 0^{+}} \frac{t^{\alpha \theta}}{1+\delta /(1-\alpha \theta) t}\left\|u\left(t, u_{0}\right)\right\|_{X^{1+\theta}}=0, \quad 0<\theta<\gamma(\varepsilon) .
$$

In fact, by Lemma 4.9, we have

$$
\begin{aligned}
& \frac{t^{\alpha \theta}}{1+\delta /(1-\alpha \theta) t}\left\|u\left(t, u_{0}\right)\right\|_{X^{1+\theta}} \\
& \leq \frac{t^{\alpha \theta}}{1+\delta /(1-\alpha \theta) t}\left\|R_{\alpha, \delta}(t) u_{0}\right\|_{X^{1+\theta}}+M c \mathbf{B}_{\varepsilon}^{\theta}(\xi) \\
& \times\left(\nu(t) t^{\alpha \gamma(\varepsilon)-\xi}+\left(\sup _{s \in(0, t]} \frac{s^{\alpha \varepsilon}}{1+\delta /(1-\alpha \varepsilon) s}\left\|u\left(s, u_{0}\right)\right\|_{X^{1+\varepsilon}}\right)^{\rho} t^{\alpha(\gamma(\varepsilon)-\rho \varepsilon)}\right. \\
& \left.\frac{(1+\delta /(1-\alpha \varepsilon) t)^{\rho}}{1+\delta /(1-\alpha \theta) t}\right) \\
& \leq \frac{t^{\alpha \theta}}{1+\delta /(1-\alpha \theta) t}\left\|R_{\alpha, \delta}(t) u_{0}\right\|_{X^{1+\theta}}+M c \mathbf{B}_{\varepsilon}^{\theta}(\xi) \nu(t)
\end{aligned}
$$




$$
\begin{array}{r}
+M c \mathbf{B}_{\varepsilon}^{\theta}(\xi) \mu^{\rho-1} \sup _{s \in(0, t]} \frac{s^{\alpha \varepsilon}}{1+\delta /(1-\alpha \varepsilon) s}\left\|u\left(s, u_{0}\right)\right\|_{X^{1+\varepsilon}} \\
\frac{(1+\delta /(1-\alpha \varepsilon) t)^{\rho}}{1+\delta /(1-\alpha \theta) t},
\end{array}
$$

where we used that $t \in\left(0, T_{0}\right], T_{0} \leq 1, \alpha \gamma(\varepsilon) \geq \xi$ and $\gamma(\varepsilon) \geq \rho \varepsilon$. The first and second terms on the right hand side of the above inequality go to 0 as $t \rightarrow 0^{+}$by Lemma 4.8 and $\lim _{t \rightarrow 0^{+}} \nu(t)=0$. On the other hand, taking $\theta=\varepsilon$ in the above inequality, we obtain

$$
\begin{aligned}
\frac{t^{\alpha \varepsilon}}{1+\delta /(1-\alpha \varepsilon) t}\left\|u\left(t, u_{0}\right)\right\|_{X^{1+\varepsilon}} \leq & \frac{t^{\alpha \varepsilon}}{1+\delta /(1-\alpha \varepsilon) t}\left\|R_{\alpha, \delta}(t) u_{0}\right\|_{X^{1+\varepsilon}} \\
& +M c \mathbf{B}_{\varepsilon}^{\varepsilon} \nu(t) \\
& +\frac{1}{4} \sup _{s \in(0, t]} \frac{s^{\alpha \varepsilon}}{1+\delta /(1-\alpha \varepsilon) s}\left\|u\left(s, u_{0}\right)\right\|_{X^{1+\varepsilon}}
\end{aligned}
$$

where we used that $\left(1+\delta /(1-\alpha \varepsilon) T_{0}\right)^{\rho-1} \leq 2$. Therefore, by Lemma 4.8 and $\lim _{t \rightarrow 0^{+}} \nu(t)=0$, we obtain

$$
\begin{aligned}
& \sup _{s \in(0, t]} \frac{s^{\alpha \varepsilon}}{1+\delta /(1-\alpha \varepsilon) s}\left\|u\left(s, u_{0}\right)\right\|_{X^{1+\varepsilon}} \\
&\left.\leq \frac{4}{3}\left(\sup _{s \in(0, t]} \frac{s^{\alpha \varepsilon}}{1+\delta /(1-\alpha \varepsilon) s} \| R_{\alpha, \delta}(s) u_{0}\right) \|_{X^{1+\varepsilon}}+M c \mathbf{B}_{\varepsilon}^{\varepsilon} \nu(t)\right) \longrightarrow 0, \\
& t \longrightarrow 0^{+} .
\end{aligned}
$$

This implies that

$$
\lim _{t \rightarrow 0^{+}} \frac{t^{\alpha \theta}}{1+\delta /(1-\alpha \theta) t}\left\|u\left(t, u_{0}\right)\right\|_{X^{1+\theta}}=0, \quad 0<\theta<\gamma(\varepsilon) .
$$

From Lemma 4.9, it follows that

$$
\begin{aligned}
& \left\|u\left(t, u_{0}\right)-u_{0}\right\|_{X^{1}} \\
& \leq\left\|R_{\alpha, \delta}(t) u_{0}-u_{0}\right\|_{X^{1}} \\
& \quad+M c \mathbf{B}_{\varepsilon}^{0}(\xi)\left(\nu(t)+\left(\sup _{s \in(0, t]} \frac{s^{\alpha \varepsilon}}{1+\delta /(1-\alpha \varepsilon) s}\left\|u\left(s, u_{0}\right)\right\|_{X^{1+\varepsilon}}\right)^{\rho}\right. \\
& \left.\frac{(1+\delta /(1-\alpha \varepsilon) t)^{\rho}}{1+\delta t}\right)
\end{aligned}
$$


as $t \rightarrow 0^{+}$, using $u_{0} \in X^{1}$, the strong continuity of $\left(R_{\alpha, \delta}(t)\right)_{t \geq 0}$, $\lim _{t \rightarrow 0^{+}} \nu(t)=0$ and the analysis of the previous step of the proof. Thus, $u\left(\cdot, u_{0}\right)$ is the unique $\varepsilon$-regular mild solution starting at $u_{0}$ in $K\left(T_{0}\right)$. This solution will be the $K$-solution starting at $u_{0}$.

Finally, let $u_{0}, w_{0} \in B_{X^{1}}\left(v_{0}, r\right)$ be given. By Lemmas 4.8 and 4.10, we obtain the inequality

$$
\begin{aligned}
& \frac{t^{\alpha \theta}}{1+\delta /(1-\alpha \theta) t}\left\|u\left(t, u_{0}\right)-u\left(t, w_{0}\right)\right\|_{X^{1+\theta}} \\
& \quad \leq M\left\|u_{0}-v_{0}\right\|_{X^{1}}+M c \mathbf{B}_{\varepsilon}^{\theta}(\zeta+\alpha \varepsilon) \\
& \quad \times\left(\nu(t) t^{\alpha(\gamma(\varepsilon)-\theta)-\zeta} \frac{1+\delta /(1-\alpha \varepsilon) t}{1+\delta /(1-\alpha \theta) t}+2 \mu^{\rho-1} t^{\alpha(\gamma(\varepsilon)+\varepsilon-\theta-\rho \varepsilon)}\right. \\
&
\end{aligned}
$$

If $\theta=\varepsilon$, we get

$$
\begin{aligned}
& \frac{t^{\alpha \varepsilon}}{1+\delta /(1-\alpha \varepsilon) t}\left\|u\left(t, u_{0}\right)-u\left(t, w_{0}\right)\right\|_{X^{1+\theta}} \\
& \quad \leq M\left\|u_{0}-v_{0}\right\|_{X^{1}}+\frac{3}{4} \\
& \quad \times \sup _{0<s \leq T_{0}} \frac{s^{\alpha \varepsilon}}{1+\delta /(1-\alpha \varepsilon) s}\left\|u\left(s, u_{0}\right)-u\left(s, w_{0}\right)\right\|_{X^{1+\varepsilon}},
\end{aligned}
$$

where we used the choice of $T_{0}, \gamma(\varepsilon) \geq \rho \varepsilon$ and $\alpha(\gamma(\varepsilon)-\varepsilon)-\zeta \geq 0$. Therefore,

$$
\sup _{0<s \leq T_{0}} \frac{s^{\alpha \varepsilon}}{1+\delta /(1-\alpha \varepsilon) s}\left\|u\left(s, u_{0}\right)-u\left(s, w_{0}\right)\right\|_{X^{1+\varepsilon}} \leq 4 M\left\|u_{0}-v_{0}\right\|_{X^{1}} .
$$

Let $0 \leq \theta \leq \theta_{0}<\gamma(\varepsilon)+\varepsilon-\rho \varepsilon$. Then, we have

$$
\begin{aligned}
& \frac{t^{\alpha \theta}}{1+\delta /(1-\alpha \theta) t}\left\|u\left(t, u_{0}\right)-u\left(t, w_{0}\right)\right\|_{X^{1+\theta}} \\
& \quad \leq M\left\|u_{0}-v_{0}\right\|_{X^{1}}+4 M^{2} c \mathbf{B}_{\varepsilon}^{\theta}(\zeta+\alpha \varepsilon) \\
& \quad \times\left(\nu(t) t^{\alpha(\gamma(\varepsilon)-\theta)-\zeta} \frac{1+\delta /(1-\alpha \varepsilon) t}{1+\delta /(1-\alpha \theta) t}+2 \mu^{\rho-1} t^{\alpha(\gamma(\varepsilon)+\varepsilon-\theta-\rho \varepsilon)}\right.
\end{aligned}
$$


$\leq C\left(\theta_{0}\right)\left\|u_{0}-w_{0}\right\|_{X^{1}}$,

$$
\left.\frac{(1+\delta /(1-\alpha \varepsilon) t)^{\rho}}{1+\delta /(1-\alpha \theta) t}\right)\left\|u_{0}-w_{0}\right\|_{X^{1}}
$$

where

$$
\begin{gathered}
C\left(\theta_{0}\right)=M\left(1+4 M \sup _{\substack{t \in\left[0, T_{0}\right] \\
\theta \in\left[0, \theta_{0}\right]}} c \mathbf{B}_{\varepsilon}^{\theta}(\zeta+\alpha \varepsilon)\left(\nu(t) t^{\alpha(\gamma(\varepsilon)-\theta)-\zeta} \frac{1+\delta /(1-\alpha \varepsilon) t}{1+\delta /(1-\alpha \theta) t}\right.\right. \\
\left.\left.2 \mu^{\rho-1} t^{\alpha(\gamma(\varepsilon)+\varepsilon-\theta-\rho \varepsilon)} \frac{(1+\delta /(1-\alpha \varepsilon) t)^{\rho}}{1+\delta /(1-\alpha \theta) t}\right)\right) .
\end{gathered}
$$

This concludes the proof.

If the non-linearity $f$ is independent of the time, then we can rewrite Theorem 4.11 in the following way.

Corollary 4.12. Let $f$ be an $\varepsilon$-regular map relative to the pair $\left(X^{1}, X\right)$ for some $\varepsilon>0$ and $v_{0} \in X^{1}$. Then, there are constants $r, T_{0}>0$ such that, for any $u_{0} \in B_{X^{1}}\left(v_{0}, r\right)$, there is a continuous function $u\left(\cdot, u_{0}\right):\left[0, T_{0}\right] \rightarrow X^{1}$ satisfying $u\left(0, u_{0}\right)=u_{0}$ and which is an $\varepsilon$-regular mild solution to (4.3). This solution satisfies

$$
\begin{gathered}
u\left(\cdot, u_{0}\right) \in C\left(\left(0, T_{0}\right], X^{1+\theta}\right), \quad 0 \leq \theta<\gamma(\varepsilon), \\
\lim _{t \rightarrow 0^{+}} \frac{t^{\alpha \theta}}{1+\delta /(1-\alpha \theta) t}\left\|u\left(t, u_{0}\right)\right\|_{X^{1+\theta}}=0, \quad 0<\theta<\gamma(\varepsilon) .
\end{gathered}
$$

Moreover, for each $\theta_{0}<\gamma(\varepsilon)+\varepsilon-\rho \varepsilon$, there exists a $C>0$ such that, if $u_{0}, w_{0} \in B_{X^{1}}\left(v_{0}, r\right)$, then

$$
\begin{gathered}
\frac{t^{\alpha \theta}}{1+\delta /(1-\alpha \theta) t}\left\|u\left(t, u_{0}\right)-u\left(t, w_{0}\right)\right\|_{X^{1+\theta}} \leq C\left\|u_{0}-w_{0}\right\|_{X^{1}}, \\
t \in\left[0, T_{0}\right], \quad 0 \leq \theta \leq \theta_{0} .
\end{gathered}
$$

We finish this section with an application to our results.

Example 4.13. Let $\Omega \subset \mathbb{R}^{N}$ be a bounded domain with smooth boundary. Let $0<\alpha<1$ and $\rho>1$ be given. We consider the problem: 


$$
\begin{cases}u=1 /(\Gamma(\alpha)) \int_{0}^{t}(t-s)^{\alpha-1} e^{-\delta(t-s)} & \\ \quad \times\left[\Delta u+u|u|^{\rho-1}\right] d s+u_{0} & \text { in }(0, T) \times \Omega, \\ u(0)=u_{0} & \text { in } \Omega, \\ u=0 & \text { on } \partial \Omega .\end{cases}
$$

The abstract formulation of (4.5) is given by

$$
u(t)=\int_{0}^{t} a(t-s)\left[A u(s)+f(s, u(s)] d s+u_{0}, \quad t \geq 0,\right.
$$

where

and

$$
a(t)=\frac{t^{\alpha-1} e^{-\delta t}}{\Gamma(\alpha)},
$$

$$
\begin{gathered}
f(u)=u|u|^{\rho-1} \\
A=\Delta \quad \text { on } E_{q}^{0}:=L^{q}(\Omega),
\end{gathered}
$$

where $q=[N(\rho-1)] / 2$ with the domain

$$
D(A)=W^{2, q}(\Omega) \cap W_{0}^{1, q}(\Omega) .
$$

We denote the fractional power spaces associated to $A$ by $\left\{E_{q}^{\beta}\right\}_{\beta \in \mathbb{R}}$. Let $A_{\beta}$ be the realization of $A$ in $E_{q}^{\beta}$. Then, it is well known that

$$
A_{\beta}: D\left(A_{\beta}\right)=E_{q}^{\beta+1} \subset E_{q}^{\beta} \longrightarrow E_{q}^{\beta}
$$

is a sectorial operator. Define

$$
X_{q}^{\beta}:=E_{q}^{\beta-1}, \quad \beta \in \mathbb{R} .
$$

Let

$$
f: X_{q}^{\gamma(\epsilon)} \longrightarrow X_{q}^{1+\epsilon}
$$

be defined by $f(u)=u|u|^{\rho-1}$. If $1<q<N /(N-2)$, then, by [2, Lemma 8], we have that $f$ is an $\epsilon$-regular map relative to $\left(X_{q}^{1}, X_{q}^{0}\right)$ for

$$
0<\epsilon_{0}(q)<\epsilon<\frac{N}{N+2 q},
$$

with

$$
\epsilon_{0}(q)=\frac{N}{N+2 q}\left(1-\frac{N}{2}\left(1-\frac{1}{q}\right)\right)
$$

and $\gamma(\epsilon)=\rho \epsilon$. 
Let $u_{0} \in L^{q}(\Omega)$ be given. From Corollary 4.12, we can conclude that there exists an $\epsilon$-regular mild solution to Problem (4.5), starting at $u_{0}$, for any $\epsilon \in\left(\epsilon_{0}(q), N /(N+2 q)\right)$. Moreover, for any $0<\theta<\gamma(\epsilon)$, this solution satisfies

$$
\frac{t^{\alpha \theta}}{1+\delta /(1-\alpha \theta) t}\left\|u\left(t, u_{0}\right)\right\|_{X^{1+\theta}} \longrightarrow 0 \quad \text { as } t \rightarrow 0^{+}
$$

and

$$
\begin{gathered}
\frac{t^{\alpha \theta}}{1+\delta /(1-\alpha \theta) t}\left\|u\left(t, u_{0}\right)-u\left(t, w_{0}\right)\right\|_{X^{1+\theta}} \leq C\left\|u_{0}-w_{0}\right\|_{L^{q}(\Omega)}, \\
t \in\left[0, T_{0}\left(u_{0}, w_{0}\right)\right]
\end{gathered}
$$

where we have used that $X_{q}^{1}=E_{q}^{0}=L^{q}(\Omega)$.

\section{REFERENCES}

1. L. Abadias, C. Lizama and P.J. Miana, Sharp extensions and algebraic properties for solutions families of vector-valued differential equations, Banach J. Math. Anal. 10 (2016), 169-208.

2. J.M. Arrieta and A.N. Carvalho, Abstract parabolic problems with critical nonlinearities and applications to Navier-Stokes and heat equations, Trans. Amer. Math. Soc. 352 (1999), 285-310.

3. E. Bajlekova, The abstract Cauchy problem for the fractional evolution equation, Fract. Calc. Appl. Anal. 1 (1998), 255-270.

4. H. Brezis and T. Cazenave, A nonlinear heat equation with singular initial data, J. Anal. Math. 68 (1996), 277-304. 2005.

5. T. Burton, Volterra Integral and differential equations, Elsevier, Amsterdam,

6. Ph. Clément and G. Da Prato, Existence and regularity results for an integral equation with infinite delay in a Banach space, Int. Eq. Oper. Th. 11 (1988), 480500 .

7. Ph. Clément and E. Mitidieri, Qualitative properties of solutions of Volterra equations in Banach spaces, Israel J. Math. 64 (1988), 1-24.

8. B. De Andrade, A.N. Carvalho, P.M. Carvalho-Neto and P. Marín-Rubio, Semilinear fractional differential equations: Global solutions, critical nonlinearities and comparison results, Topol. Meth. Nonlin. Anal. 45 (2015), 439-467.

9. K.J. Engel and R. Nagel, One-Parameter semigroups for linear evolution equations, Grad. Texts Math. 194, Springer, New York, 2000.

10. A. Friedman, On integral equations of Volterra type, J. Anal. Math. 11 (1963), 381-413. 
11. G. Gripenberg, On Volterra equations of the first kind, Int. Eq. Oper. Th. 3 (1980), 473-488.

12. G. Gripenberg, S.-O. Londen and O. Staffans, Volterra integral and functional equations, in , Encyclopedia of mathematics and its applications 34, Cambridge University Press, Cambridge, 1990.

13. B.H. Guswanto and T. Suzuki, Existence and uniqueness of mild solutions for fractional semilinear differential equations, Electr. J. Diff. Eq. 168 (2015), 1-16.

14. M. Haase, The functional calculus for sectorial operators, in Operator theory: Advances and applications 169, Birkhauser, Basel, 2006.

15. A. Jawahdou, Mild solutions of functional semilinear evolution Volterra integrodifferential equations on an unbounded interval, Nonlin. Anal. 74 (2011), 7325-7332.

16. A.A.Kilbas, H.M. Srivastava and J.J. Trujillo, Theory and applications of fractional differential equations, Elsevier, Amsterdam, 2006.

17. J. Kemppainen, J. Siljander, V. Vergara and R. Zacher, Decay estimates for time-fractional and other non-local in time subdiffusion equations in $\mathbb{R}^{d}$, Math. Ann. 366 (2016), 941-979.

18. K. Li and J. Peng, Fractional resolvents and fractional evolution equations, Appl. Math. Lett. 25 (2012), 808-812.

19. C. Lizama, Regularized solutions for abstract Volterra equations, J. Math. Anal. Appl. 243 (2000), 278-292.

20. C. Lizama and G. N'Guérékata, Mild solutions for abstract fractional differential equations, Appl. Anal. 92 (2013), 1731-1754.

21. Z.D. Mei, J.G. Peng and Y. Zhang, A characteristic of fractional resolvents, Fract. Calc. Appl. Anal. 16 (2013), 777-790.

22. Z. Ouyang, Existence and uniqueness of the solutions for a class of nonlinear fractional order partial differential equations with delay, Comp. Math. Appl. 61 (2011), 860-870.

23. J. Prüss, Evolutionary integral equations and applications, Birkhauser/Springer, Basel, 2012.

24. C. Roberts, Characterizing the blow-up solutions for nonlinear Volterra integral equations, in Proceedings of the Second World Congress of Nonlinear Analysts, Part 2, Nonlin. Anal. 30 (1997), 923-933.

25. F.B. Weissler, Semilinear evolution equations in Banach spaces, J. Funct. Anal. 32 (1979), 277-296.

26. Local existence and nonexistence for semilinear parabolic equations in $L_{p}$, Indiana Univ. Math. J. 29 (1980), 79-102. 
Universidad de Santiago de Chile, Departamento de Matemática y Ciencia de la Computación, Casilla 307, Correo 2, Santiago, Chile

Email address: luciano.abadias@gmail.com

Universidad del Norte, Departamento de Matemáticas y Estadística, BarRANQUilla, Colombia

Email address: ealvareze@uninorte.edu.co

Universidad de Santiago de Chile, Departamento de Matemática y Ciencia de la Computación, Casilla 307, Correo 2, Santiago, Chile

Email address: carlos.lizama@usach.cl 